\title{
Susceptibility to experimental infection of the invertebrate locusts (Schistocerca gregaria) with the apicomplexan parasite Neospora caninum
}

Neuropathogenesis is a feature of Neospora caninum infection. In order to explore this in the absence of acquired host immunity to the parasite, we have tested infection in locusts (Schistocerca gregaria). We show for the first time that locusts are permissive to intrahemocoel infection with $N$. caninum tachyzoites. This was characterized by alteration in body weight, fecal output, hemoparasitemia, and sickness-related behavior. Infected locusts exhibited progressive signs of sickness leading to mortality. Also, N. caninum showed neuropathogenic affinity, induced histological changes in the brain and was able to replicate in the brain of infected locusts. Fatty acid (FA) profiling analysis of the brains by gas chromatography and multi-variate prediction models discriminated with high accuracy (98\%) between the FA profiles of the infected and control locusts. DNA microarray gene expression profiling distinguished infected from control S. gregaria brain tissues on the basis of distinct differentially-expressed genes. These data indicate that locusts are permissible to infection with $N$. caninum and that the parasite retains its tropism for neural tissues in the invertebrate host. Locusts may facilitate preclinical testing of interventional strategies to inhibit the growth of $N$. caninum tachyzoites. Further studies on how $N$. caninum brings about changes in locust brain tissue are now warranted. 
1 Mamdowh M. Alkurashi ${ }^{1,2}$, Sean T. May ${ }^{3}$, Kenny Kong ${ }^{4}$, Jaume Bacardit ${ }^{5}$, David Haig $2 \quad$, Hany M. Elsheikha ${ }^{1 *}$

$3{ }^{1}$ School of Veterinary Medicine and Science, University of Nottingham, Sutton Bonington 4 Campus, Leicestershire LE12 5RD, UK

$5{ }^{2}$ Animal Production Department, College of Food and Agricultural Sciences, King Saud 6 University, Riyadh 11451, Saudi Arabia

$7{ }^{3}$ Nottingham Arabidopsis Stock Centre, Division of Plant and Crop Sciences, School of 8 Biosciences, University of Nottingham, Leicestershire, UK

$9 \quad{ }^{4}$ School of Physics and Astronomy, University of Nottingham, UK

$10{ }^{5}$ School of Computing Science, Newcastle University, Newcastle-upon-Tyne, NE1 7RU, UK

\section{$11 *$ Corresponding author.}

12 Tel.: +44 1159516445; fax: +44 1159516440;

13 E-mail address: hany.elsheikha@nottingham.ac.uk (H. Elsheikha). 


\section{INTRODUCTION}

15 Infection by the apicomplexan protozoan Neospora caninum is an important cause of

16 infertility and abortion in cattle and neuromuscular disorders in dogs (Barber and Trees, 1996;

17 Vonlaufen et al., 2002; Dubey et al., 2007; Innes, 2007). With no effective vaccine and limited

18 anti-parasitic treatments available, $N$. caninum poses a pressing economical and animal health

19 threat. Limited understanding of molecular mechanisms of cerebral neosporosis in hosts

20 contributes to the lack of effective treatments. Hence, increased knowledge of the molecular

21 events that lead to brain damage is essential.

22 Neospora caninum is an obligatory intracellular organism that has the capacity to invade a

23 wide range of cells both in vitro and in vivo (Vonlaufen et al., 2002). Various infection hosts have

24 been used to understand $N$. caninum neuropathogenesis including in vitro studies using cell lines

25 or organotypic cultures, which provided key information about host cell invasion by N. caninum

26 and the cellular events that occur during intracellular parasite proliferation (Vonlaufen et al.,

27 2002; Dubey et al., 2007). Studies of N. caninum infection and the resulting neuropathologies

28 have also been observed for in vivo vertebrate models, including: cats, mice, rats, gerbils and

29 monkeys (Dubey, 1999; Collantes-Fernández et al., 2004; Pinheiro et al., 2006; Bartley et al.,

30 2007; Reichel and Ellis, 2009). These models suggested several molecules and mechanisms key

31 to the establishment and progression of infection (Collantes-Fernández et al., 2002; Vonlaufen et

32 al., 2002), and allowed potential vaccinations for neosporosis to be assessed (Bartley et al., 33 2007).

34 Vertebrate animals are expensive to maintain and large numbers are usually needed to yield 35 statistically valid data. Thus, understanding the molecular determinants of $N$. caninum brain 36 infection in a cost effective and productive organ culture or whole animal system would be 37 advantageous. Virtually nothing is known about invertebrate host responses to infection with $N$. 38 caninum. Invertebrate hosts have proven valuable in understanding aspects of host-parasite 
39 interactions (Siddiqui et al., 2011). For example, the protist, Acanthamoeba shows commonality

40 in targeting the blood-brain barrier of locusts and humans, suggesting that the invertebrate locust

41 can mimic human Acanthamoeba encephalitis and reveal a spectrum of host-pathogen

42 interactions (Mortazavi et al., 2009; 2010). Examples such as these support the use of a locust

43 system to study protozoan infection and to investigate virulence determinants in vivo.

44 Insects rely on an innate immune system for their protection against infection, so the use of

45 an insect model is relevant in the study of $N$. caninum infection, the control of which has

46 dependency on the innate immune system (McAllister et al., 2000; Bartley et al., 2013). The

47 evolutionary conservation of several aspects of the innate immune response between

48 invertebrates and mammals (Hoffmann et al., 1999; Lemaitre and Hoffmann, 2007) and the fact

49 that $N$. caninum utilizes similar virulence factors in phylogenetically distant hosts studied so far

50 makes the use of a simple invertebrate host, such as $S$. gregaria an attractive one for studying

51 parasite pathogenesis. Indeed, locusts have already been used to answer questions regarding host-

52 pathogen interactions in other microbial and protozoan systems, such as Acanthamoeba

53 (Mortazavi et al., 2009; 2010) as mentioned above and also in studies with neuropathogenic $E$.

54 coli K1 (Khan and Goldsworthy, 2007; Mokri-Moayyed et al., 2008).

55 In this study, we examined the susceptibility of a non-mammalian host for infection with $N$.

56 caninum. Adult locusts, $S$. gregaria are found to be susceptible to infection with $N$. caninum

57 tachyzoites given via the intra-hemocoel route of inoculation and can be used as surrogate hosts

58 for $N$. caninum. The parasite infection exhibited strict neurotropism, and resulted in brain

59 pathology and molecular changes associated with sickness and ultimately death of the locusts.

60 MATERIALS AN DMETHODS

61 Research ethics statement 
62 This study was reviewed by the University of Nottingham (UK) School of Veterinary

63 Medicine and Science (SVMS) Ethical Review Committee. The Committee reviews all research

64 studies involving School personnel and is chaired by Professor David Haig. The committee

65 passed this study as good to proceed, not requiring any further ethical review as it involved

66 invertebrates. FELASA guidelines as outlined in 'principles and practice in ethical review of

67 animal experiments across Europe (2005)' and UK guidelines on the use of invertebrates in

68 research were followed.

\section{Parasite strain and growth conditions}

70 Neospora caninum (Liverpool strain) was cultured in human brain microvascular endothelial

71 cells (HBMECs) originally obtained from ScienCell Research Laboratories (Elsheikha et al.,

72 2013). HMBECs were used at passage 18 and were grown in T75 flasks (Corning) in tissue-

73 culture medium composed of RPMI-1640 medium containing L-glutamine and sodium

74 bicarbonate, and supplemented with 20\% (v/v) heat inactivated FBS, $1 \mathrm{mM}$ Sodium Pyruvate, 1\%

75 MEM non-essential amino acids, 1\% MEM vitamins and 2\% penicillin/streptomycin. Cells were

76 maintained in an incubator in a humidified atmosphere at $37^{\circ} \mathrm{C}$ and $5 \% \mathrm{CO} 2$. Once confluent $(\sim 3$

77 days), cells were trypsinized using trypsin-EDTA (Invitrogen, GIBCO, UK). N. caninum

78 tachyzoites were harvested from cultured cells, purified as described (18), and counted using a

79 haemocytometer and diluted to the desired concentration, $10^{3}, 10^{4}, 10^{5}$, and $10^{6}$ tachyzoites 80 suspended in $20 \mu 1$ culture medium, for inoculation.

\section{Locusts}

82 Desert locusts, Schistocerca gregaria of gregarious morphotype, were obtained from a local

83 breeder (www.livefoodsdirect.co.uk). Locusts were kept in a large glass cage for not less than one

84 week after arrival to acclimatize and reduce any stress associated with transportation. Adult 
85 locusts were used throughout the study to ensure a fully developed innate immune system. Each

86 group of ten locusts (5 males and 5 females) were housed in separate plastic "critter cages" with

87 ventilation slats in the lid and solid plastic bottoms to enable feces collection. They were fed

88 daily with fresh grass and wheat seedlings supplemented with bran. Water was provided ad

89 libitum in petri dishes to increase hemolymph volume. The "critter cages" were kept on the bench

90 in a controlled environment at $25^{\circ} \mathrm{C}$ and $65 \%$ humidity with a 12 -hr light cycle. During the

91 parasite exposure period, housing and manipulation of locusts was performed within a biological

92 safety cabinet (class II) inside an ABSL2+ containment area.

\section{Infection conditions}

94 Locusts were randomized into five groups (G1 to G5), with 10 locusts per group and were

95 housed in separate "critter cages" to prevent cross-cage contamination after parasite challenge. To

96 establish a suitable dose for $N$. caninum tachyzoites, $10^{3}, 10^{4}, 10^{5}$, or $10^{6}$ tachyzoites were

97 inoculated in a total volume of $20 \mu 1$ culture medium into the hemocoel of locusts in groups G1 to

98 G4, respectively. Locusts in G5 were mock-inoculated with medium only and used as controls.

99 An additional control group was included (i.e. locusts which were not injected but incubated

100 under the same conditions as infected locusts). Injection of the test materials was achieved using

101 a 26g Terumo ${ }^{\circledR}$ Neolus hypodermic needle fitted into plastic pipette tips. Inocula were injected

102 into the hemocoel by inserting the needle horizontally into the inter-segmental membrane at the

103 last two abdominal segments using a pipetter, followed by gentle stretching of the locust's

104 abdomen to ensure an even distribution of parasites within the hemocoel (Fig. S1). Individual

105 locusts were identified by numbering on the underside of the thoracic cuticle using a permanent

106 marker pen. Locusts were observed daily for the duration of the experiment. 
107 Survival

108 Locusts were assessed for mortality twice daily and any dead locusts were removed to

109 prevent cannibalism. Post mortem analysis was performed within the safety cabinet. Control

110 locusts were sacrificed by making an incision behind the head at the end of the experiment.

111 Survival data was analyzed using the Gehan-Breslow-Wilcoxon test, with death as the primary

112 variable using GraphPad Prism version 5.00 for Windows (GraphPad Software, San Diego, CA) .

113 Significance between survival curves was assessed using the Log-rank (Mantel-Cox) test.

\section{Morbidity after parasite challenge}

115 To assess the effect of $N$. caninum infection on the behavior of the locusts, a range of 116 parameters were evaluated. These include the following: (i) The number of times the insect was

117 inactive. (2) The number of walking bouts (a walk was defined as an unbroken period of 118 locomotion of more than half a body length). (3) The number of times leg movements occurred.

119 (4) The number of times antennal movements occurred. (5) The number of times grooming 120 occurred. (6) The number of times wing-fanning occurred. (7) The number of times jumping 121 occurred. This experiment was divided into two 15 min periods. During the first 15 min period, 122 individual behavioral variables, chosen after preliminary observations, were registered every 10 123 seconds for locusts in each cage.

\section{Detection of hemoparasitemia}

125 To determine whether locusts developed parasitemia, hemolymph was collected from one 126 locust per group $3 \mathrm{hr}$ pi and daily thereafter for three consecutive days. Hemolymph was 127 collected from locusts by inserting a 1-10 $\mu 1$ pipette tip fitted into a pipette-aid into the arthrodial 128 membrane at the base of the legs (Fig. S2). At least $20 \mu 1$ of hemolymph was aspirated from each 
129 locust (depending on the hydration of the locust) and a smear was made on a clean microscope 130 glass slide and checked for the presence of $N$. caninum tachyzoites under X40 magnification.

\section{Body weight}

132 To assess the effect of $N$. caninum infection on food consumption locusts were weighed prior 133 to infection and daily for the duration of the experiment. The body weight changes were 134 calculated as a percentage of initial body weight. Each locust was transferred from the cage 135 within a closed 50-ml falcon tube, weighed on a weighing balance and returned back to the cage 136 within the same falcon tube. Results are presented as means \pm SEM from three independent 137 experiments.

\section{Fecal output}

139 Another indirect measure was used to assess the effect of exposure to N. caninum on food 140 consumption where the output of fecal pellets was determined on a daily basis. Fecal pellets 141 produced per cage over a $24 \mathrm{hr}$ period were separated from non-fecal materials and weighed. This

142 figure was divided by the number of surviving locusts to give an average of fecal output per 143 locust.

\section{Detection of N. caninum in locusts' brain}

145 In a second experiment one group of five locusts were inoculated with $10^{4}$ tachyzoites and 146 another group was sham inoculated with medium only. One locust from each of the infected and 147 control groups was sacrificed daily and samples from the brains of each locust were collected and 148 preserved in 5\% paraformaldehyde for histology or 70\% ethanol for genetic characterization. For 149 histological analysis, samples were fixed for at least $24 \mathrm{hr}$ before they were sectioned and stained 150 with hematoxylin and eosin. Also, PCR-DNA sequencing was used to document the presence of 
151 growing parasites in the brain. Genomic DNA was extracted from the locust's brain using a

152 DNeasy Blood and Tissue Kit (Qiagen Inc.) following the Animal Tissues Spin Protocol supplied

153 by the manufacturer. The DNA was eluted in $50 \mu \mathrm{l}$ of kit elution buffer and stored at $-20^{\circ} \mathrm{C}$.

154 Concentration and purity of DNA in sample extracts were checked by using a Thermo Scientific

155 NanoDrop ${ }^{\mathrm{TM}} 1000$ Spectrophotometer, prior to PCR amplification. The two most commonly

156 species-specific primers used to diagnose $N$. caninum, primers Np6 plus

157 (5'CTCGCCAGTCAACCTACGTCTTCT3') $\quad$ Np21 plus

158 (5'CCCAGTGCGTCCAATCCTGTAAC3'), were used to amplify approximately 337-350-bp

159 fragment of the pNC-5 gene, which is commonly used to identify $N$. caninum infection (Muller et

160 al., 1996). pNC-5 has been shown in previous studies to be $N$. caninum species-specific and a

161 variable region (Kaufmann et al., 1996; Yamage et al., 1996). Hence, this gene might be a useful

162 genetic marker for discrimination between different $N$. caninum isolates. Approximately $50 \mathrm{ng}$ of

163 genomic DNA was amplified using $40-\mu 1$ reaction mixture containing $2 \mu 1$ of extracted DNA, $20 \mu 1$

164 of Biomix (Bioline, UK), $17 \mu$ l of nuclease-free water (Fisher Scientific), and $0.5 \mu l(\sim 10 \mathrm{pmol})$ of

165 each forward and reverse oligonucleotide primer (Eurofins). Biomix BioMix ${ }^{\mathrm{TM}}$ is a complete,

166 ready-to-use, $2 \mathrm{x}$ reaction mix containing Taq DNA polymerase. It is used to perform PCR assays

167 of numerous genomic and cDNA templates by only adding water, template and primers. All

168 amplifications were carried out in triplicates using the Bioer Xp Cycler. The PCR cycling

169 conditions consisted of an initial denaturation for $5 \mathrm{~min}$ at $94^{\circ} \mathrm{C}$ and then 40 cycles of $94^{\circ} \mathrm{C}$ for 1

$170 \mathrm{~min}, 63^{\circ} \mathrm{C}$ for $1 \mathrm{~min}$, and extension at $74^{\circ} \mathrm{C}$ for $3.5 \mathrm{~min}$, followed by a final extension at $72^{\circ} \mathrm{C}$ for

$17110 \mathrm{~min}$. A negative control (nuclease-free water instead of DNA) and a positive control (DNA

172 from cultured-derived $N$. caninum) were included in each run. The quality and specificity of all

173 the amplification products were assessed by $2 \%$ agarose gel electrophoresis followed by staining

174 with ethidium bromide. Individual PCR product bands were visualized under ultraviolet light.

175 Amplicons were purified using a QIAquick PCR Purification Kit (Qiagne) according to the 
176 manufacturer's instructions. Cleaned-up products were sequenced bidirectionally using the same

177 PCR oligonucleotide primers used in initial amplification using a commercial service (Source

178 Biosciences Inc., Nottingham, England). To gain insight into the anatomic sites of N. caninum

179 distribution and replication, we detected N. caninum DNA in several different body tissues of

180 locusts using the same PCR assay described above.

\section{Recovery of infecting parasite strain}

182 For this experiment 12 freshly dead locusts were randomly selected from groups which have

183 been infected with $10^{3}, 10^{4}, 10^{5}$, or $10^{6}$ tachyzoites (three locusts per group). At 5,10 or 17 day PI

184 brain was obtained from one locust from each group (i.e. a total of four brains, one from each

185 group), and were dissected under aseptic conditions for parasite isolation. Brains were washed

186 twice in RPMI medium, divided into small pieces by scissors and homogenized by pipetting up

187 and down gently 10 times through a 1-ml pipette tip, followed by passing the homogenate

188 through an 18-gauge needle and syringe. The resulting homogenate from four brains at each of

189 the time point mentioned above were pooled and resuspended in $6 \mathrm{ml}$ of RPMI medium. The 6

$190 \mathrm{ml}$ homogenate from the pooled four brains $(1 \mathrm{ml} /$ well) was overlaid on healthy HBMECs seeded

191 in a 6-well culture plate (Nunc Inc., Denmark) and topped up by a second $\mathrm{ml}$ of fresh RPMI

192 medium, and plate was incubated at $37^{\circ} \mathrm{C}$. After $24 \mathrm{hr}$ the culture medium was decanted, and $2 \mathrm{ml}$

193 of fresh RPMI medium was added to each well. One 6-well culture plate was seeded at 5, 10 or

19417 day PI. All plates were incubated in a humidified atmosphere at $37^{\circ} \mathrm{C}$, and were examined

195 daily for signs of parasite growth within cultured cells by inverted light microscopy.

Characterization of locust's brain-derived isolate

197 We tested if the passage of $N$. caninum in a non-natural host has favoured parasite 198 phenotypic or genotypic changes. The species identity of the locust-derived $N$. caninum isolate 
199 was confirmed and characterised in comparison to the original isolate. Tachyzoites from each

200 isolate were subjected to a range of phenotypic and genetic characterisation methods. Firstly,

201 immunofluorescence staining of tissue culture infected with each isolate was performed as

202 described previously (Alkurashi et al., 2011). Secondly, for transmission electron microscopy

203 (TEM), HBMEC culture containing tachyzoites originated from each isolates were fixed in $2 \%$

204 glutaraldehyde in $0.2 \mathrm{M}$ sodium cacodylate buffer ( $\mathrm{pH} 7.4)$ for $3 \mathrm{hr}$ and were post-fixed for $1 \mathrm{hr}$

205 in 1\% osmium tetroxide in the same buffer; they were then dehydrated in acetone and embedded

206 in epoxy resin. Finally, ultrathin $(80 \mathrm{~nm})$ sections were cut with a Leica EM UC6 microtome, and

207 contrasted with uranyl acetate and lead citrate. Sections were examined with a Phillips Morgagni

208268 (FEI company, Hillsboro, OR) transmission electron microscope operating at $80 \mathrm{kV}$. Digital

209 images were recorded with a MegaViewIII digital camera operated with iTEM software

210 (Olympus Soft Imaging Systems, Germany). The Adobe Photoshop CS4 digital photography

211 editing program was used for additional processing. Thirdly, purified parasite preparation from

212 each isolate was used for PCR-sequencing analysis using species-specific primers Np21plus-

213 Np6plus which anneal into the Nc5 region of $N$. caninum as described above. Fourthly, chemical

214 profile of tachyzoites from each isolate was obtained by using Confocal Raman spectroscopy,

215 scanned over at $0.5 \mu \mathrm{m}$ step sizes. At least 13 tachyzoites from each isolate were studied. A k-

216 means clustering method was used to separate the spectra from the tachyzoites and substrate, and

217 then, the spectra from tachyzoites of both isolates were used to do principle component analysis

218 (PCA) to identify chemical differences. The measurements were performed using $785 \mathrm{~nm}$ laser,

219 and at $3 \mathrm{sec}$ integration time for every spectrum. Fifthly, approximately $10^{5}$ tachyzoites derived

220 from one locust-derived isolate were used for subsequent inoculation into five new locusts to

221 assess the effect of passage into a non-natural host on the parasite's ability to retain its

222 neurotropism. Locusts were monitored daily for 3 weeks for mortality and sickness behavior. All

223 experiments were performed three independent times. 


\section{Gas Chromatography (GC) profiling of brain lipids}

225 Twenty five locusts were randomly allocated to five groups each with five locusts. Each

226 locust was inoculated with $10^{4} \mathrm{~N}$. caninum tachyzoites; this dose was chosen because it produces

227 clinical illness in the infected locusts. Negative-control locusts were injected with $20 \mu 1$ of

228 medium. One day after infection and daily thereafter, five locusts from each group (infected or

229 control) were sacrificed by cervical dislocation and their brains were dissected within the safety

230 cabinet, providing five replicates per group. Brain samples were immediately frozen in liquid

231 nitrogen and homogenised using a gentleMACSTM closed homogeniser (Milteny Biotec Ltd.,

232 Surrey, UK). Homogenised tissues were subjected to lipid extraction as described previously

233 (Folch et al., 1957). Samples were transesterified by the method of Christie (1982) and modified

234 by Chouinard et al. (1999). The fatty acid methyl esters were injected (split ratio 50:1) into gas

235 chromatograph (GC 6890; Agilent technologies Ltd, Stockport, UK). Separation of fatty acid

236 methyl esters was performed with a Varian CP-Sil 88 (Crawford ScientificTM Ltd., Strathaven,

237 UK) capillary column with hydrogen as carrier gas. The fatty acid methyl esters were identified

238 by comparing the retention times with a fatty acid methyl esters standard mixture (Sigma-Aldrich

239 Co LLC, Gillingham, UK) and the area percentage in moles were used for the statistical analysis.

240 A total of 37 fatty acids were analyzed in this study, and included: saturated fatty acids: C4:0,

241 C6:0, C8:0, C10:0, C11:0, C12:0, C14:0, C15:0, C16:0, C17:0, C18:0, C20:0, C21:0, C22:0,

242 C23:0, C24:0. Monosaturated fatty acids: C14:1, C15:1, C16:1, C17:1, C20:1, C24:1.

243 Polysaturated fatty acid- Omega (n)-3: C18:3n3, C20:3n3, C20:5n3, C22:6n3. Omega (n)-6:

244 C18:2n6t, C18:2n6, C20:3n6, C20:4n6. Omega (n)-9: C18:1n9t, C18:1n9C, C20:1n9.

245 Data obtained from the brain phospholipid composition was filtered of poorly measured fatty

246 acids (defined as fatty acids with percentiles below $0.01 \%$ ). Afterwards, the data were evaluated

247 using SPSS 16.0 (SSPS $®$ Inc. Chicago, USA) using a general linear model. The significance of 
248 each fatty acid was assessed using p-values corrected for multiple comparisons using the

249 Bonferroni method. All fatty acids that obtained a p-value $<0.05$ were kept for further analysis.

250 Multi-variate prediction models were constructed to determine if it was possible to

251 discriminate between the parasite-infected samples and the controls. Specifically we have used 252 our own machine learning method called BioHEL (Bacardit et al., 2009), that has been 253 successfully applied in the past for the analysis of lipidomics data (Fainberg et al., 2012) and 254 transcriptomics (Bassel et al., 2011; Glaab et al., 2012). The prediction capacity of BioHEL on 255 the locust samples was estimated using the robust leave-one-out cross-validation procedure, in 256 which a prediction model is build using all but one of the samples, and the remaining sample is 257 used to test the validity of the model. This procedure is repeated 50 times, each time using a 258 different sample to test the models. The prediction capacity of the system is estimated as the 259 percentage of correctly predicted samples.

260 Finally, in order to identify which are the combinations of fatty acids presenting the highest 261 prediction capacity, new prediction models were generated where each model used only two, 262 three or four of the fatty acids, testing all possible combinations. Again, leave-one-out cross263 validation was used to estimate the prediction capacity of these reduced panels of fatty acids.

264 Detection of differential gene expression using the drosophila gene chip

265 In a new experiment three locusts were each inoculated with $10^{4} \mathrm{~N}$. caninum tachyzoites.

266 Three locusts were sham inoculated with RPMI medium and used as controls. One day after 267 infection, locust brains were dissected, frozen in liquid nitrogen, transferred to a pre-chilled (with 268 liquid nitrogen) mortar and ground with a pestle to a very fine powder with liquid nitrogen. The 269 powdered samples were transferred to $2 \mathrm{ml}$ Eppendorf microtubes and used for extraction of total 270 RNA. Total RNA was extracted from $50 \mathrm{mg}$ sample powder using the QIAGEN RNeasy Mini Kit 271 (QIAGEN, Germantown, MD). To verify the quality of the RNA, the yield and purity were 
272 determined spectrophotometrically (NanoDrop, Wilmington, DE) and with an Agilent 2100

273 Bioanalyzer using RNA 6000 Nano kits (Agilent Technologies, Palo Alto, CA). A whole

274 transcript drosophila array (GeneChip Drosophila Genome 2.0 Array, Affymetrix, Santa Clara,

275 USA) was assessed for the study of gene expression in locust. The hybridization of heterologous

276 (non-specific) nucleic acids onto arrays designed for model-organisms have been shown to be a

277 viable genomic resource for estimating gene expression in many non-model organisms (Lai et al.,

278 2014). This approach enables the investigation of gene expression in locusts in the absence of a

279 fully sequenced genome.

280 Analysis of the cross-species hybridization data was performed as follows: Biotin labeled 281 locust genomic DNA was hybridized to a drosophila_2 chip (Affymetrix, Santa Clara, USA) and 282 used as described previously (Lai et al., 2014) to convert a standard drosophila_2 Chip Definition 283 File (CDF file, Affymetrix, Santa Clara, USA) into a series of bespoke locust.cdf files. The newly 284 derived CDF file having the maximum number of filtered probes, whilst retaining all probe-sets, 285 was selected and subsequently used in all downstream analysis. Statistical analysis of mRNA 286 expression profiling was done by analysis of variance (ANOVA) using Partek Genomics Suite 2876.5 (Partek Incorporated, MO). Results were significant if fold change was more than 1.5 or less 288 than -1.5 , with $\mathrm{P}<0.05$. Cluster analyses and principal component analysis were conducted with 289 Partek default settings.

290 RESULTS

291 Clinical outcomes of infection

292 Mortality rate 
293 Locusts died gradually as the infection progressed over the course of the experiment which

294 lasted up to 27 days PI. Locusts infected with larger number of $N$. caninum tachyzoites showed

295 less survival time, where all locusts in G1, G2, G3 and G4 died by day 25, 23, 21 and 20 PI,

296 respectively (Fig. 1). Compared with control locusts, locusts receiving N. caninum infection

297 appeared to have statistically significant higher mortality [control versus (G1, $p=0.0008),(\mathrm{G} 2$;

$298 p=0.0007),(\mathrm{G} 3 ; p=0.0004)$, or $(\mathrm{G} 4 ; p=0.0047)]$. The mortality rate didn't follow a dose-

299 dependent manner, despite the increase in inoculation dose. Two locusts from the negative

300 (uninfected) control group and one locust from the environmental control group had died during

301 the course of the experiment, but their death was not due to infection with $N$. caninum based on a 302 negative PCR result for brains of those two locusts.

303 Effects of infection on sickness behavior

304 Assessment of various behavioral parameters revealed significant inter-locust variability that 305 did not correlate with differences in the dose of infection. For example, variability in behavioral 306 parameters in individual locusts reared in the same cage varied by as much as 5-fold, which 307 precluded any statistical analysis to be performed. The source of this variability among locusts is 308 not understood despite the standardization of age, food, handling, and rearing conditions. Despite 309 this variability we observed abnormal behavior in locusts two days after infection that increased 310 over time. Most of the locusts exhibited a sluggish mobility one day before death and some 311 showed dark blue discoloration on the day of death.

\section{Hemoparasitemia}

313 Tachyzoites of $N$. caninum were observed in the hemolymph collected from all infected 314 locusts up to $48 \mathrm{hr}$ pi. Subsequently, no evidence of the parasites was obtained in hemolymph 
315 from any locust. Collection of $20 \mu 1$ of hemolymph from each locust did not seem to have any

316 effect on the activity or weight of the infected or control locusts.

\section{Effects of infection on body weight}

318 Infection with $N$. caninum did not cause a remarkable reduction in the body weight (Fig. 2).

319 However, the distribution of data over the experiment shows that body weights decrease

320 gradually in infected groups. At_the lowest parasite challenge_(G1 infected with $10^{3}$ tachyzoites),

321 the locusts did not gain or lose weight, and there was no significant difference between locusts

322 from the sham-infected or environmental groups. However, there was a statistically significant

323 difference in weight change between control locusts and $N$. caninum-infected locusts in the other

324 groups $(\mathrm{G} 2, p=0.0024 ; \mathrm{G} 3, p<0.0001 ; \mathrm{G} 4, p=0.0012)$. The calculation of the average body

325 weight of each group could not be performed beyond day 15 pi because only a few locusts were

326 left in infected groups, which precluded direct quantitative comparison between different groups.

\section{Fecal output}

328 A slight increase in fecal output was detected in infected locusts 1 day PI, followed by 329 progressive decrease in the subsequent days especially in locusts given the higher doses. But, a 330 dose-response reduction in the fecal output was not detected (Fig. 3). No difference was detected 331 in any of the tested clinical parameters of infection between either control or infected male and 332 female locusts (data not shown). However, there was some variability in the response of locusts, 333 not gender-specific, from experiment to experiment.

\section{Evidence for N. caninum parasites in the brain}


335

336

337

338

339

340

341 inflammatory loads observed between locusts' brains from different infection groups. Given the

342 significant mortality with $10^{3}$ it is interesting that surviving animals have normal body weight.

343 Apart from the limited pathological changes that were detected in surviving locusts, there is no

344 evidence to indicate that surviving locusts from this group were able to control the infection

345 compared to those who died. However, this might be attributed to the variability in individual

346 response of locusts to infection. The parasite has not been detected in any other organ of any

347 locust examined.

\section{DNA extraction and PCR amplification}

In all brain tissue of infected locusts, the PCR products were successfully amplified to the expected 337-bp parasite-specific fragment, which was found to correspond to the targeted $N$. caninum sequence within the Nc5 gene as confirmed by sequencing analysis. Positive PCR products from locusts' brains were obtained from day one to day five PI (Fig. 5) and were found to slightly increase as infection progressed over time. Even though end-point PCR can detect and quantify specific DNA sequences it is commonly used as a semi-quantitative method, and thus results should be confirmed by quantitative reverse transcription-quantitative PCR (RT-qPCR). No genetic evidence for the presence of tachyzoites was detected in the brain of control locusts for up to 14 day PI. PCR was also used to determine parasite distribution to other body sites (fat 
358 body and muscle). The parasite DNA was not detected in any of the examined non-brain tissues.

359 Likewise, there was no evidence for the presence of the parasite in feces.

\section{Recovery of viable parasites from locusts' brain}

361 Using a cell culture bioassay, viable $N$. caninum tachyzoites were successfully isolated from 362 pooled brain homogenates of locusts that were infected with $10^{3}, 10^{4}, 10^{5}$, or $10^{6}$ tachyzoites at

363 day 10 and 17 PI. Out of the 18 inoculated wells only two wells at 10 and 17 day PI yielded new 364 tachyzoites. Recovered isolates were maintained in vitro via serial passages in cultured 365 endothelial cells for four months before being stored in liquid nitrogen. No significant difference 366 was observed in the rate of proliferation and growth kinetic of both locust-derived isolates and 367 the culture-derived strain of N. caninum (data not shown).

368 Characterization of N. caninum isolate derived from locust' brain

369 Immunofluorescent (Fig. S3) and TEM (Fig. S4) analyses showed that tachyzoites derived 370 from locust's brain are morphologically-identical to tachyzoites of the culture-derived original 371 isolate. Locust-derived tachyzoites retained the same phenotypic and genetic characteristics of 372 tachyzoites of the original $N$. caninum isolate. DNA sequences from $N$. caninum tachyzoites of 373 each isolate was identical, and a consensus sequence obtained from both isolates showed 100\% 374 sequence homology to N. caninum sequence accession number AY497045 in GenBank database.

375 It was important to find out if the passage of the parasite through the locust had induced any 376 alteration in the phenotype of the parasite. Chemical profiling using Confocal Raman 377 Spectroscopy technique (Fig. 6) and principle component analysis (Fig. 7) revealed minor, non378 significant differences between the two N. caninum isolates. Locust-brain-derived N. caninum 379 isolate was used to infect locusts to test their neuropathogenic capacity. In vivo passage in 380 locust's brain did not result in alteration in neuropathogenicity. One of the locust-adapted isolates 
381 induced brain infection in $100 \%$ of five newly infected locusts, which had detectable parasite

382 burdens in the brain at different time points pi as evidenced by PCR. But, no further attempt was

383 made to re-isolate the parasites from these locusts and use for infection again.

384 Lipid profiling

385 In an effort to understand the mechanisms underlying the complex relationships between the

386 host locust and parasite infection, we compared the fatty acid profile of brain from infected

387 locusts to that for their control counterparts. Among the 37 fatty acids analysed in this study,

388 results were consistently obtained from all tested samples for only seven FAs and included:

389 saturated fatty acids: C14:0, C16:0, C18:0; monounsaturated fatty acids: C16:1; polysaturated

390 fatty acid-Omega (n)-3: C18:3n3. Omega (n)-6: C18:2n6, C18:1n9C. Fig. 8 shows a heatmap

391 visualising the relationships of these fatty acids to the 50 samples ( 25 infected, 25 controls). The

392 heatmap suggests that no individual fatty acid is strongly associated to any of the two groups.

393 Nonetheless, our analysis using the BioHEL machine learning algorithm reveals that we can

394 create multi-variate prediction models that can assign samples to treatments with very high

395 accuracy. The results of the data analysis are summarised in the supplementary file (Table S1).

396 When the prediction models use the seven FAs we can correctly predict 98\% (all but one) of the

397 samples. To check if an even more reduced panel of fatty acids presents high discriminative

398 power we tested all combinations of two, three and four fatty acids. The best groups of four

399 [Palmitic acid methyl ester (C16:0), Palmitoleic acid methyl ester (C16:1), Linoleic acid methyl

400 ester (C18:2n6c), and Linolenic acid methyl ester (C18:3n3)] and three [C16:1, C18:2n6c and

401 C18:3n3] FAs managed to still give an accuracy of $98 \%$. The best group of two fatty acids (C16:1

402 C18:2n6c) reduced its accuracy to $94 \%$ (mis-classifying three samples).

403 Gene expression microarray data 


\section{PeerJ Reviewing Manuscript}

404 To obtain a broader understanding of the effects of the N. caninum on S. gregaria adults, we

405 examined the parasite impact on host gene expression using Affymetrix DNA microarray and

406 Cross Species Hybridisation (CSH) analysis. Since N. caninum infection induced neurological

407 injuries in locusts, it was important to test whether $N$. caninum infection had altered gene 408 expression within locust brains. The analysis was designed to gain further understanding of the 409 mechanisms for $N$. caninum neuropathy, and in particular, of the genes responsible for the 410 capacity of $N$. caninum to establish brain infection. We used an established (Lai et al, 2014) cross 411 species hybridisation (CSH) approach, which applied genomic DNA pre-filtration to identify

412 conserved probes operationally useful between phylogenetically disparate species; in this case 413 allowing us to use Drosophila Gene Chips to assay locust RNA. Samples of RNA from brains of 414 mock- and N. caninum-infected locusts were labeled and hybridised to Affymetrix Gene Chip 415 Drosophila Genome 2.0 Arrays. Data were analyzed using Partek Genomics Suite Version 6.4. Of 416 the 28,593 transcripts represented on the GeneChip, approximately 18,500 were expressed in the

$417 N$. caninum-infected locust's brain. PCA quality control analysis shows that the uninfected and 418 infected samples were biologically noisy but generally well separated (Fig. S5). The subsequent 419 analysis of differential expression was constrained by $\geq 1.5$-fold difference in expression where 420 Fig. 9 shows a graphical representation (volcano plot) of the differential analysis suggesting that 421 our cutoff is somewhat conservative and indicates several significantly differential genes with

422 low fold-change that are not discussed further in this report. It is clear that there are substantial 423 differences in gene expression between infected and control samples. Our chosen fold-change

424 boundaries indicated that 17 transcripts changed significantly $(P<0.05$ with FDR filtering) 425 between the uninfected and $N$. caninum-infected locusts. Of these transcripts, 11 increased $>1.5$ 426 fold and 6 decreased $>-1.5$-fold (Table 1). Of the up-regulated transcripts to which a function 427 could be assigned, one transcript was associated with signal transduction and autophagy. Other 428 functional categories that included down-regulated transcripts were developmental processes and 
429 metabolism. The identification of these DE transcripts could provide a pipeline for promising 430 targets to test subsequently in mice.

431 Hierarchical clustering and PCA identifies distinctive molecular phenotypes in infected vs. 432 control locusts

433 DNA microarray expression profiling demonstrated significant differences in gene

434 expression between infected and control locusts. From the 17 statistically_-significant probes that 435 represented multiple genes, a hierarchical clustering heat map revealed two distinctive patterns 436 that closely correlated with the two study groups (Fig. 10).

\section{DISCUSSION}

438 Previously, N. caninum was known to circulate between mammalian and bird species (Mineo 439 et al., 2011) only and considered limited by a "species barrier," which might depend on 440 differences in the parasite virulence traits with regard to host animal physiology. Herein, we 441 demonstrate for the first time that a strain of $N$. caninum (Nc-Liverpool) was able to infect the 442 invertebrate desert locust with induction of signs of illness and death. The progression to a lethal 443 N. caninum infection in this non-natural host may result from the cumulative deleterious effects 444 of both direct and indirect consequences of parasite infection that lead to accelerated destruction 445 and compromised host immune regenerative capacity. $N$. caninum did not elicit a typical 446 mortality dose response curve after intra-hemocoel infection and the reason for this is not known.

447 However, the lack of dose-dependent mortality can be explained by the existence of a threshold 448 effect that occurs with relatively moderate number of parasites $\left(10^{3}\right.$ to $\left.10^{4}\right)$, after which large 449 differences in parasite number make little difference. It is also possible that -immune response 450 might have been elicited against this virulent strain to control parasite dissemination and interfere 451 with the fatal outcome following increasing infectious challenge doses of the parasite. Doses 
452 from $10^{5}$ to $10^{6}$ parasites might seem high for the establishment of an infection for downstream

453 analysis. On the other hand, infection with less number of parasites ( $<1000$ tachyzoites) didn't

454 seem to cause any effect on the locust's sickness, mortality, or body weight. For these reasons, a

455 dose of $10^{4}$ was chosen for subsequent experiments, due to the reasonably long mean time of

456 death after infection, which allows more time for data collection but is pathogenic enough to

457 determine the effect of infection.

458 Also, there was a reduction of body weight and fecal output in response to infection, but not

459 in a dose-dependent manner. This is most likely due to the use of pooled rather than individual

460 fecal samples. The possibility that this was caused by changes in the diet of locusts was ruled out

461 by dietary standardization. Parasites disappeared from the hemolymph of the locusts after 2 day

462 pi. Infected locusts showed reduced mobility and sickness. The possible risk due to the

463 manipulation of the locusts during weighing or collection of hemolymph was ruled out because

464 these handling issues didn't seem to affect the control groups. Mock-infected and environmental

465 control locusts, apart from the death of one locust per group, appeared normal. The study of

466 locusts' behaviour highlighted the difficulties associated with the behavioural assay. Many of the

467 tested variables are not independent of one another (e.g. walking vs leg movement vs inactivity).

468 It could have been more useful to analyze the amount of time locusts spent doing each activity,

469 rather than the number of bouts. Also, the assay has not been standardized to control for the time

470 of day. Locusts show a certain amount of diurnal variation in activity levels that may have

471 contributed to the variability of the assay results._Following the intra-hemocoel inoculation of $N$.

472 caninum into locusts, $N$. caninum migrated to the brain within $24 \mathrm{hr}$ accompanied by induced

473 histological alterations and infection-specific inflammatory responses-, suggesting that the insect

474 immune response is recognising and responding to the parasites. However, because S. gregaria is

475 not a natural host of $N$. caninum in the field, it unlikely that locust is able to mount a N. caninum- 
476 specific response. It remains to be investigated if histo-pathological changes are $N$. caninum-

477 specific or inflammatory response to the parasite invasion.

478 Previous studies in mice (Collantes-Fernández et al., 2004), wild rodents (Ferroglio et al.,

479 2007), dogs (Peters et al., 2000), or calves (Kritzner et al., 2002) showed that the parasite DNA 480 and/or lesions could be detected in the brain as well as other tissues, such as muscle, heart, liver,

481 spleen, lung, and pancreas. In the present study, although tissue tropism was evident by the 482 presence of parasite DNA in the brain (Fig. 5), it was interesting to note that all examined non483 brain tissues did not show evidence for the presence of the parasite, suggesting the brain as the 484 preferred target organ in experimental N. caninum infection in locusts. An alternative explanation 485 is that the parasite is strictly neurotropic and its absence in other tissues is due to the lack of 486 significant neuronal tissues in other organs in the locust, unlike in vertebrates. These findings are 487 different to an earlier report where the protist Acanthamoeba organisms were able to invade 488 different organs of the infected locusts and did not exhibit any tissue-specific preference 489 (Mortazavi et al., 2010).

490 Two developmental stages, an acute phase and a chronic (cystic) phase, are known to occur 491 during $N$. caninum infection in the vertebrate animals (Peters et al., 2000; Kritzner et al., 2002; 492 Collantes-Fernández et al., 2004; Ferroglio et al., 2007). In the present study, only acute tissue 493 invasion by tachyzoites, commonly associated with acute phase of infection with $N$. caninum, 494 was able to be established in the locusts. One might argue that the demonstration of the 495 occurrence of the two life cycle forms of $N$. caninum within the locust might have made it more 496 representative to the natural (or vertebrate) host. However, this is a new in vivo approach to 497 culturing $N$. caninum and the first report of an experimental establishment of $N$. caninum 498 infection in an invertebrate host. The availability of this experimental invertebrate host opens an 499 avenue for $N$. caninum research in defining determinants of the parasite virulence, and host roles 500 in disease pathogenesis along with a reduction of the number of vertebrate animals used in 
501 research. The use of the invertebrate locust has proven valuable to discriminate molecules 502 participating from both sides of the host-parasite interaction (Siddiqui et al., 2011). For example, 503 neuropathogenic E. coli $\mathrm{K} 1$ pathogenesis within both locust and mammalian systems exhibited 504 remarkable similarities in producing bacteremia leading to bacterial invasion of the central 505 nervous system and has been shown to be dependent upon several common established virulence 506 factors, such as LPS, OmpA, FimH, and CNF1 (Khan and Goldsworthy, 2007; Mokri-Moayyed 507 et al., 2008). Assuming a correlation between the virulence of $N$. caninum in locusts and in 508 vertebrates $N$. caninum-induced killing of the locusts can be exploited as an assay system to 509 screen for neuropathogenesis virulence-attenuated mutants of $N$. caninum or in preclinical testing 510 of interventional strategies to interfere with the growth of $N$. caninum tachyzoites.

511 An important result was the recovery of viable $N$. caninum tachyzoites from the brain of 512 experimentally infected $S$. gregaria for up to 17 days PI. The isolation of a viable $N$. caninum 513 isolate from the brain of infected S. gregaria provided an opportunity to examine whether

514 passage of the parasites through a totally non-natural host induces phenotypic and/or genotypic 515 changes in these parasites. Thus, the phenotype, genetic and chemical profile of $N$. caninum 516 isolate derived from S. gregaria and the original isolate were compared. S. gregaria-derived 517 isolate exhibited similar growth pattern to the original isolate (unpublished data). The two 518 isolates were subjected to nucleotide sequence analysis of the Nc5 gene and compared to each 519 other and to previously described $N$. caninum sequences available in Genbank database. PCR520 sequencing analysis revealed that both parasite isolates are identical at least with respect to this

521 gene. Due to the multi-copy nature of Nc5 gene direct sequencing of PCR products of this gene 522 may not that informative. Alternatively, cloning prior to sequencing followed by careful 523 alignments of cloned sequences is a more sensitive approach to distinguish between all variants 524 of the gene, and can enable the recovery of information useful for estimation of genetic diversity 525 between $N$. caninum isolates. Minor, non-significant differences were found between the two 
526 isolates by using Raman spectroscopy. A large amount of spectral data obtained from the two

527 isolates analysed by multivariate analysis did not reveal two distinct clusters, identifying only a

528 few chemical metabolites that were different between the two parasite isolates (Fig. 7).

529 One of the objectives of this study was to test whether passage of the parasite in a non-

530 natural host can affect the parasite's virulence and neurotropism. Such evidence was acquired by

531 injecting parasites derived from brain of $S$. gregaria, via a cell culture bioassay, into new $S$.

532 gregaria hosts. The locust derived isolate resulted in successful infection of $100 \%$ of the newly

533 infected hosts, indicating that this parasite has the ability to adapt to changing physiological

534 environment and retained its capacity to invade the brain in newly infected S. gregaria. Studying

535 the implications of serial passages of $N$. caninum in $S$. gregaria on the parasite's virulent activity

536 and the evolution of the infection in the locusts are among the future directions of the work. Host

537 adaptation is a well-known strategy to improve the infectivity of a pathogen in a non-natural host.

538 However, it risks the concomitant disadvantage of biasing the natural tropism of the pathogen.

539 Interestingly, this alteration in the tropism of N. caninum did not happen in the locust. These

540 results indicate that the current definition of the species barrier, which has been based on host

541 specificity, needs to be reassessed for $N$. caninum. The possibility of $N$. caninum crossing the

542 species barrier and infecting hosts of diverse phylogenetic backgrounds will have important

543 evolutionary implications.

544 Fundamental to the understanding of the complex and multifaceted interactions between the

545 parasite and locusts' brain was the study of global locust's response to infection by using the

546 high-throughput approaches, such as lipidomic and transcriptomic, validated for use in other

547 species. Measuring the types and the abundance of lipids in brain from healthy and infected

548 locusts using GC has provided a baseline lipid profile in S. gregaria brain and the subsequent

549 response to $N$. caninum infection. The multi-variate prediction models generated using BioHEL

550 were able to discriminate with good accuracy (98\%) between the FA profiles of the infected and 
551 control samples (Fig. 8). Moreover, by restricting the models to use only small subsets of two,

552 three and four fatty acids we identified that the minimal subset of FAs with the highest prediction

553 capacity was that of size three (C16:1, C18:2n6c and C18:3n3). It is worth noting that $\mathrm{C} 16: 1$ and

$554 \mathrm{C} 18: 2 \mathrm{n} 6 \mathrm{c}$ was always present in the best panels of FAs of sizes two, three and four. The idea that

555 fatty acid metabolism is influenced by the Neospora represents just one example where lipidomic

556 analysis has provided new hypotheses to explore. This $N$. caninum-specific alteration in fatty

557 acids is a characteristic also observed in a previous study based on transcriptome analysis in mice

558 infected with N. caninum (Ellis et al., 2012).

559 We next sought to determine the genes specifically altered in the brain by the presence of the 560 parasite. We investigated transcriptome variations in brains of both $N$. caninum-infected $S$.

561 gregaria and non-infected control locusts at $24 \mathrm{hr}$ pi. This unbiased approach allowed us to

562 categorize genes in both infected and non-infected brain tissues whose expression was altered by

563 the presence of infection. This is the first study to show the benefit of CSH for an orthopteran ( $S$.

564 gregaria) species using a whole genome oligonucleotide microarray of the fruit fly Drosophila

565 melanogaster. In line with previous studies in mammalian (Nieto-Díaz et al., 2007) and avian

566 hosts (Crowley et al., 2009), our CSH analysis using non-vertebrate insect species did not affect

567 the reproducibility of the hybridization data from Affymetrix GeneChips. This analysis provided

568 insights into the first global transcriptional response of locusts to $N$. caninum infection. Ten

569 locust genes associated with the immune response and with a variety of cellular pathway were

570 identified as up-regulated $>1.5$-fold and 6 as down-regulated $>-1.5$-fold $24 \mathrm{hrs}$ after infection

571 (Table 1). Most of the differentially expressed genes were not annotated, so their unction is

572 unknown. However, one of the upregulated genes is Atg9, which encodes for a transmembrane

573 autophagy-related protein and has been shown to induce c-Jun N-terminal kinase (JNK) signaling

574 and autophagy in response to oxidative stress in Drosophila (Tang et al., 2013). In mammalian

575 cells, mAtg9 plays an essential role in regulating oxidative stress-induced JNK activation. One of 
576 the down-regulated genes is the pannier gene, which encodes a zinc-finger transcription factor of

577 the GATA family and is known to be involved in several developmental processes during

578 embryonic and imaginal development in Drosophila. In agreement of this finding, gene

579 expression analysis of $N$. caninum infection in mice revealed changes in the expression of genes

580 associated with mammalian development, embryogenesis and fatty acid metabolism (Ellis et al.,

581 2012). Even though few differentially expressed genes were found to be statistically significant

582 our CSH experiment provides proof-of-principle of a transcriptomics workflow for investigating

583 how locusts' brain gene expression is modulated due to infection. Transcriptomic data obtained

584 from locusts in the present study in addition to the S. gregaria expressed sequence tags (EST)

585 data from the locust CNS (Badisco et al., 2011) represent an important source of information that

586 will be instrumental in further unraveling the underlying mechanisms of brain dysfunction in

587 locusts in response to infection with $N$. caninum and other neuropathogens.

588 Transcriptome profiling analyses during $N$. caninum infection in mice have been published

589 (Ellis et al., 2010; 2012). These studies revealed major changes of gene expression patterns

590 depending on factors, such as N. caninum strain, the mouse type and time post infection. Given

591 these facts, it is not surprising to observe differences in gene expression between the invertebrate

592 locusts and the mammalian animals. Interestingly, cataloguing changes in S. gregaria host gene

593 expression in response to $N$. caninum infection identified potential molecular processes

594 associated with parasite colonization of locust's brain. By looking more closely at the biological

595 significance of some of the changes in gene expression using RT-qPCR and extending over a time

596 course of the infection it can be possible to establish further the significance of the differential

597 expression results obtained in our transcriptomic analysis. The alteration of gene expression may

598 be a response to $N$. caninum infection per se or may be a component of disease pathogenesis.

599 Subsequent studies should investigate the influence of $N$. caninum infection versus virulence on 600 gene expression. To determine which of the identified genes correlated with virulence rather than 
601 just $N$. caninum infection; various $N$. caninum isolates, such as the naturally attenuated Nc-

602 Nowra, and NCts-8 (relatively avirulent) and its wild type (NC-1) isolate with different virulence

603 and cystogenic capability should be utilized.

604 Invertebrates confer additional advantages compared to vertebrate models, since they are less

605 expensive, easy to obtain, maintain and handle experimentally and can facilitate the development

606 and testing of new treatment/preventive strategies. The importance of invertebrate animals for the

607 study of fungal pathogenesis has been reviewed recently (Desalermos et al., 2012). Studies over

608 the past few years have demonstrated that the $S$. gregaria can be used as an alternative to

609 mammalian models of infection; particularly in order to investigate host-pathogen relationship

610 (Khan and Goldsworthy, 2007; Mokri-Moayyed et al., 2008; Mortazavi et al., 2009; 2010). Our

611 study provides a new opportunity for testing the feasibility of locust as an alternative model of

612 protozoal infections. However, there are still many questions to answer, including how $N$.

613 caninum is able to reach and persist in the locust brain. Whether locusts provided a permissive

614 environment to N. caninum or the parasite possesses a high affinity to brain tissue of S. gregaria,

615 is still unknown. There are many possibilities for future studies utilizing this invertebrate host. It

616 could be interesting to assess if locust could act like paratenic host/reservoir of the infection. It is

617 important to quantify, using a quantitative PCR, the amount of parasites able to cross the "brain

618 blood barrier". Sequencing a 350pb fragment provided a preliminary evidence of the genetic

619 similarity between locust-derived and original (culture-maintained) isolates; this short segment of

620 the DNA is not enough to determine genotypic changes, more sensitive methods, such as SNP

621 analysis should be pursued in subsequent studies. Also, study of the possible change in the

622 protein expression pattern could be more informative that the Raman spectrometry analysis.

\section{Competing interests}

624 The authors declare that they have no competing interests. 


\section{Acknowledgements}

626 The authors wish to thank Professor Lord Sandy Trees for providing N. caninum (Nc-

627 Liverpool) strain, Naveed Khan for providing comments on the experimental infection of the

628 locusts and Ekramy Elmorsy for his help with some of the statistical analysis, and Pablo Fainberg

629 for his help with lipid extraction. The support of the University of Nottingham's High

630 Performance Computing cluster is greatly appreciated.

\section{References}

632 Alkurashi, M., Eastick, F.A., Kuchipudi, S.V., Rauch, C., Madouasse, A., Zhu, X.Q., Elsheikha, 633 H.M., 2011. Influence of culture medium $\mathrm{pH}$ on internalization, growth and phenotypic 634 plasticity of Neospora caninum. Vet. Parasitol. 177, 267-274.

635 Bacardit, J., Burke, E.K., Krasnogor, N., 2009. Improving the scalability of rule-based 636 evolutionary learning. Memetic Computing 1(1), 55-67.

637 Badisco, L., Huybrechts, J., Simonet, G., Verlinden, H., Marchal, E., Huybrechts, R., Schoofs, L., 638 De Loof, A., Vanden Broeck, J., 2011. Transcriptome analysis of the desert locust central nervous system: production and annotation of a Schistocerca gregaria EST Database. PLoS

641 Barber, J.S., Trees, A.J., 1996. Clinical aspects of 27 cases of neosporosis in dogs. Vet. Rec. 139, 642 439-443.

643 Bartley, P.M., Wright, S., Chianini, F., Buxton, D., Innes, E.A., 2007. Inoculation of Balb/c mice with live attenuated tachyzoites protects against a lethal challenge of Neospora caninum. Parasitology 135, 13-21.

Bartley, P.M., Katzer, F., Rocchi, M.S., Maley, S.W., Benavides, J., Nath, M., Pang, Y., Cantón, G., Thomson, J., Chianini, F., Innes, E.A. 2013. Development of maternal and foetal immune responses in cattle following experimental challenge with Neospora caninum at day 210 of gestation. Vet. Res. 44:91.

652

Bassel, G.W., Glaab, E., Marquez, J., Holdsworth, M.J., Bacardit, J., 2011. Functional network construction in Arabidopsis using rule-based machine learning on large-scale data sets. The Plant Cell 23, 3101-3116. 
653 Chouinard, P.Y., Corneau, L., Saebo, A., Bauman, D.E., 1999. Milk yield and composition during 654 abomasal infusion of conjugated linoleic acids in dairy cows. J. Dairy Sci. 82, 2737-2745.

655 Christie, W.W., 1982. A simple procedure for rapid transmethylation of glycerolipids and 656 cholesteryl esters. J. Lipid Res. 23, 1072-1075.

657 Collantes-Fernández, E., Alvarez-García, G., Pérez-Pérez, V., Pereira-Bueno, J., Ortega-Mora, 658 L.M., 2004. Characterization of pathology and parasite load in outbred and inbred mouse 659 models of chronic Neospora caninum infection. J. Parasitol. 90, 579-583.

660 Collantes-Fernández, E., Zaballos, Á., Álvarez-García, G., Ortega-Mora, L.M., 2002. 661 Quantitative detection of Neospora caninum in bovine aborted fetuses and experimentally 662 infected mice by real-time PCR. J. Clin. Microbiol. 40, 1194-1198.

663 Crowley, T.M., Haring, V.R., Burggraaf, S., Moore, R.J., 2009. Application of chicken 664 microarrays for gene expression analysis in other avian species. BMC Genomics 10(Suppl 2), $665 \mathrm{~S} 3$.

666 Desalermos, A., Fuchs, B.B., Mylonakis, E., 2012. Selecting an invertebrate model host for the 667 study of fungal pathogenesis. PLoS Pathog. 8(2), e1002451.

668 Dubey, J.P., 1999. Recent advances in Neospora and neosporosis. Vet. Parasitol. 84, 349-367.

669 Dubey, J.P., Schares, G., Ortega-Mora, L.M., 2007. Epidemiology and control of neosporosis and 670 Neospora caninum. Clin. Microbiol. Rev. 20, 323-367.

671 Ellis, J., Sinclair, D., Morrison, D., Al-Qassab, S., Springett, K., Ivens, A., 2010. Microarray 672 analyses of mouse responses to infection by Neospora caninum identifies disease associated 673 cellular pathways in the host response. Mol. Biochem. Parasitol. 174(2), 117-127.

674 Ellis, J., Goodswen, S., Kennedy, P.J., Bush, S., 2012. The core mouse response to infection by 675 Neospora caninum defined by gene set enrichment analyses. Bioinform. Biol. Insights 6, $676 \quad 187-202$.

677 Elsheikha, H.M., Mckinley, C., Elsaied, N.A., Smith, P.A., 2013. Effects of Neospora caninum 678 infection on brain microvascular endothelial cells bioenergetics. Parasit. Vectors 6, 24.

679 Fainberg, H.P., Bodley, K., Bacardit, J., Li, D., Wessely, F., Mongan, N.P., Symonds, M.E., 680 Clarke, L., Mostyn, A., 2012. Reduced neonatal mortality in Meishan piglets: A role for 681 hepatic fatty acids? PLoS ONE 7(11), e49101.

682 Ferroglio, E., Pasino, M., Romano, A., Grande, D., Pregel, P., Trisciuoglio, A., 2007. Evidence of 683 Neospora caninum DNA in wild rodents. Vet. Parasitol. 148(3-4), 346-349.

684 Folch, J., Lees, M., Sloane Stanley, G.H., 1957. A simple method for the isolation and 685 purification of total lipides from animal tissues. J. Biol. Chem. 226, 497-509. 
686 Glaab, E., Bacardit, J., Garibaldi, J.M., Krasnogor, N., 2012. Using rule-based machine learning 687 for candidate disease gene prioritization and sample classification of cancer gene expression 688 data. PLoS ONE 7, e39932.Hoffmann, J.A., Kafatos, F.C., Janeway, C.A., Ezekowitz, R.A., 689

690 Innes, E.A., 2007. The host-parasite relationship in pregnant cattle infected with Neospora 691 caninum. Parasitology 134, 1903-1910.

692 Kaufmann, H., Yamage, M. Roditi, I., Dobbelaere, D., Dubey, J. P., Holmdahl, O. J. M., Trees, 693 A., Gottstein, B., 1996. Discrimination of Neospora caninum from Toxoplasma gondii and 694 other apicomplexan parasites by hybridization and PCR. Mol. Cell. Prob. 10, 289-297.

695 Khan, N.A., Goldsworthy, G., 2007. A novel model to study virulence determinants of 696 Escherichia coli K1. Infect. Immun. 5, 5735-5739.

697 Kritzner, S., Sager, H., Blum, J., Krebber, R., Greif, G., Gottstein, B., 2002. An explorative study 698 to assess the efficacy of toltrazuril-sulfone (ponazuril) in calves experimentally infected with 699 Neospora caninum. Ann. Clin. Microbiol. Antimicrob. 18, 1-4.

700 Lai, H-M., May, S.T, Mayes, S., 2014. Pigeons: a novel GUI software for analysing and parsing 701 high density heterologous oligonucleotide microarray probe level data. microarrays, 3(1), 170223.

703 Lemaitre, B., Hoffmann, J., 2007. The host defense of Drosophila melanogaster. Annu. Rev. $704 \quad$ Immunol. 25, 697-743.

705 | McAllister MM, Bjorkman C, Anderson-Sprecher R, Rogers DG. 2000. Evidence of point-source 706 exposure to Neospora caninum and protective immunity in a herd of beef cows. J. Am. Vet. 707 Med. Assoc. 217, 881-887.

708 Mineo, T.W., Carrasco, A.O., Raso, T.F., Werther, K., Pinto, A.A., Machado, R.Z., 2011. Survey 709 for natural Neospora caninum infection in wild and captive birds. Vet Parasitol. 182(2-4), $710 \quad 352-355$.

711 Mokri-Moayyed, B., Goldsworthy, G.J., Khan, N.A., 2008. Development of a novel ex vivo 712 insect model to study virulence determinants of Escherichia coli. J. Med. Microbiol. 57, 106713110.

714 Mortazavi, P.N., Goldsworthy, G., Kirk, R., Khan, N.A., 2009. Novel model for the in vivo study 715 of central nervous system infection due to Acanthamoeba spp. (T4 genotype). J. Med. 716 Microbiol. 58(Pt 4), 503-508. 
717 Mortazavi, P.N., Goldsworthy, G., Kirk, R., Khan, N.A., 2010._Acanthamoeba produces

718 disseminated infection in locusts and traverses the locust blood-brain barrier to invade the 719 central nervous system. BMC Microbiol. 10, 186.

720 Muller, N., Zimmermann, V., Hentrich, B., Gottstein, B., 1996. Diagnosis of Neospora caninum 721 and Toxoplasma gondii infection by PCR and DNA hybridization immunoassay. J. Clin. 722 Microbiol. 34, 2850-2852.

723 Nieto-Díaz, M., Pita-Thomas, W., Nieto-Sampedro, M., 2007. Cross-species analysis of gene 724 expression in non-model mammals: reproducibility of hybridization on high density 725 oligonucleotide microarrays. BMC Genomics 8, 89.

726 Peters, M., Wagner, F., Schares, G., 2000. Canine neosporosis: clinical and pathological findings 727 and first isolation of Neospora caninum in Germany. Parasitol. Res. 86(1), 1-7.

728 Pinheiro, A.M., Costa, S.L., Freire, S.M., Almeida, M.A.O., Tardy, M., El Bacha, R., Costa, 729 M.F.D., 2006. Astroglial cells in primary culture: A valid model to study Neospora caninum 730 infection in the CNS. Vet. Immunol. Immunopathol. 113, 243-247.

731 Reichel, M.P., Ellis, J.T., 2009. Neospora caninum - How close are we to development of an 732 efficacious vaccine that prevents abortion in cattle? Int. J. Parasitol. 39, 1173-1187.

733 Siddiqui, R., Mortazavi, P., Pleass, R., Khan, N.A., 2011. Non-vertebrate models to study parasite 734 invasion of the central nervous system. Trends Parasitol. 27(1), 5-10.

735 Tang, H.W., Liao, H.M., Peng, W.H., Lin, H.R., Chen, C.H., Chen, G.C., 2013. Atg9 interacts 736 with dTRAF2/TRAF6 to regulate oxidative stress-induced JNK activation and autophagy 737 induction. Dev. Cell. 27(5), 489-503.

738 Vonlaufen, N., Gianinazzi, C., Müller, N., Simon, F., Björkman, C., Jungi, T.W., Leib, S.L., 739 Hemphill, A., 2002. Infection of organotypic slice cultures from rat central nervous tissue 740 with Neospora caninum: an alternative approach to study host-parasite interactions. Int. J. 741 Parasitol. 32, 533-542.

742 Yamage, M., Flechtner, O., Gottstein, B., 1996. Neospora caninum: specific oligonucleotide 743 primers for detection of brain "cyst" DNA of experimentally infected nude mice by the 744 polymerase chain reaction. J. Parasitol. 82, 272-279. 


\section{Figure 1 (on next page)}

Survival of locusts given various doses of Neospora caninum tachyzoites by the intrahemocoel route.

Groups (G1 to G4) of locusts ( $n=10$ ) were administered doses of $N$. caninum of $10^{3}(\mathrm{G} 1), 10^{4}$ (G2), $10^{5}(\mathrm{G} 3)$, and $10^{6}(\mathrm{G} 4)$ per locust. Control locusts were sham-inoculated with RPMI cultured medium. An environmental control group (e-group) of non-infected locusts incubated under the same conditions as other groups was also included. Survival was monitored daily after infection. Results represent average survival curve based on three independent experiments. Control versus $\mathrm{G1}(p=0.0008)$; control versus $\mathrm{G} 2(p=0.0007)$; control versus $\mathrm{G} 3$ ( $p=0.0004)$; control versus $\mathrm{G} 4(p=0.0047)$. Locusts inoculated with 10 or 100 tachyzoites did not exhibit any signs of sickness or mortality (data not shown) 


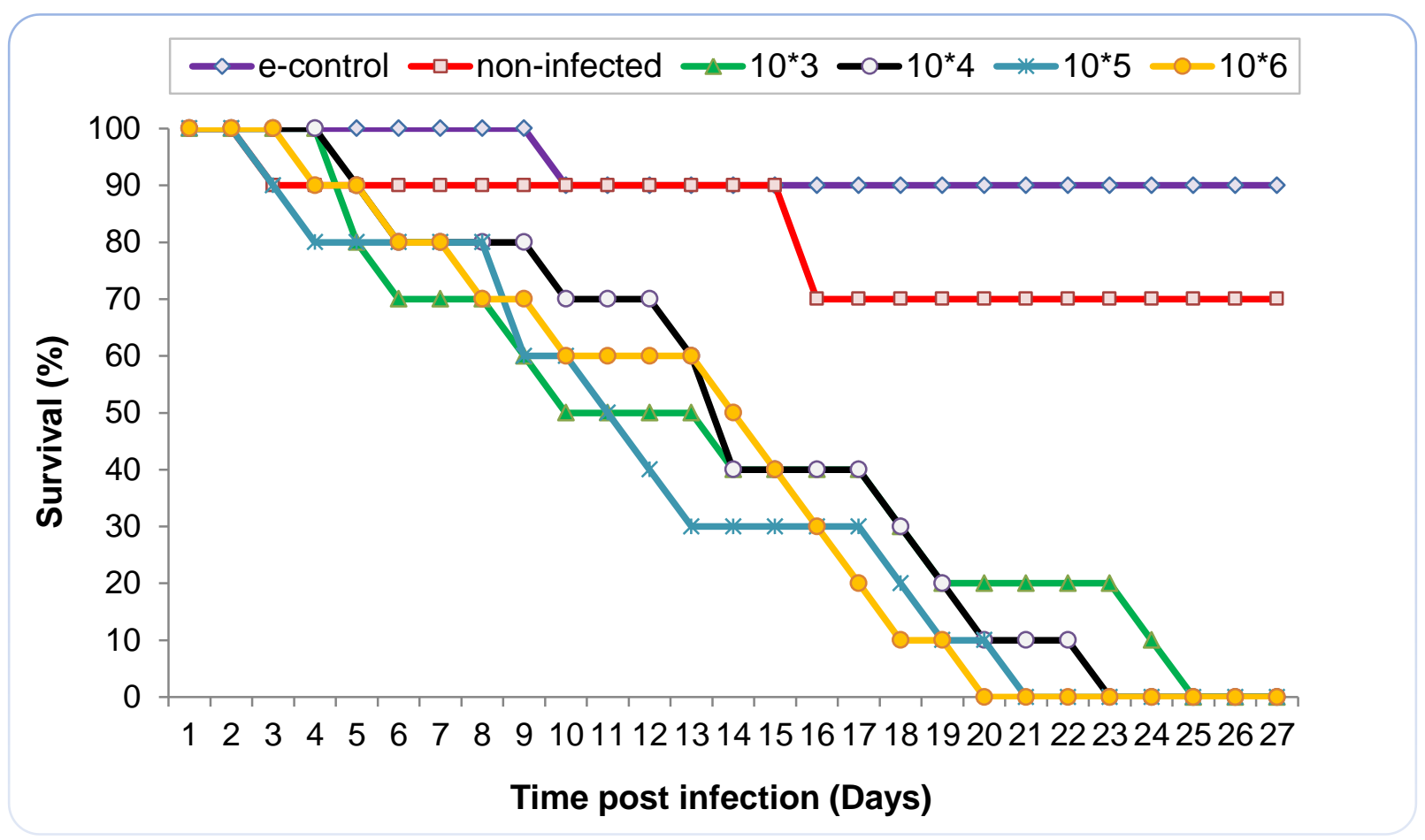




\section{Figure 2 (on next page)}

Relative body weight (BW) of Neospora caninum-infected locusts compared to controls at different time points after infection.

Shown are means \pm SEM of percent body weight change compared with initial body weight for surviving locusts at each time point. There was no significant change in the BW of control locusts and locusts in group 1 (infected with $10^{3}$ tachyzoites) along the course of the experiment, but in groups 2 and 4 , infected with $10^{4}$ and $10^{6}$, respectively, there was significant loss in weight beginning by 2 day after infection ( $p=0.0024$ and 0.0012 , respectively). In group 3 infected with $10^{5}$ the weight loss began by 3 day after infection ( $p$ $<0.0001$ ). Not all locusts completed the course of the experiment due to associated mortality. Data was compered using paired t-test ( $p$-value $<0.05$ ). 


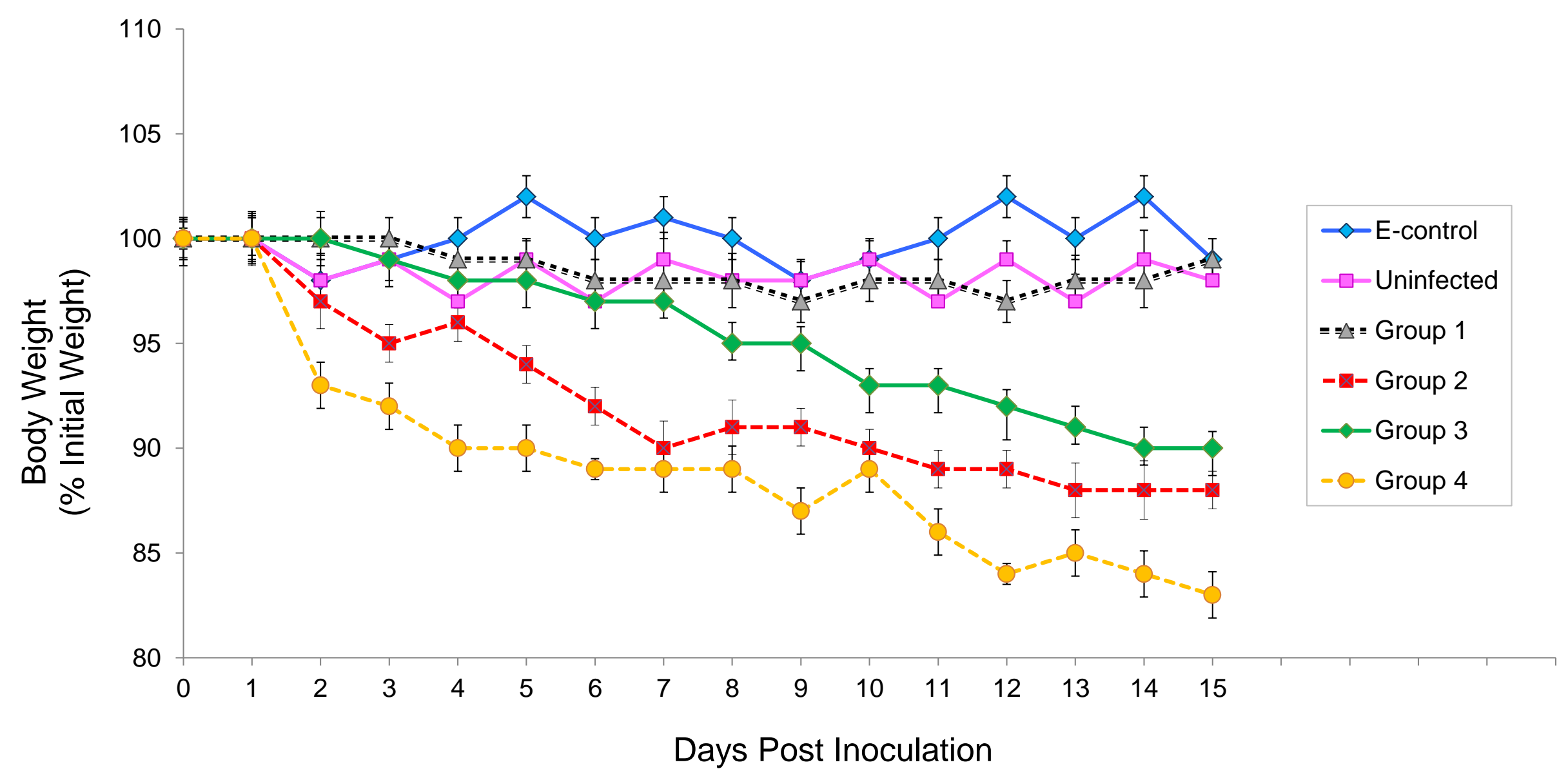




\section{Figure 3 (on next page)}

The effect of Neospora caninum infection on locust fecal output.

Besides the environmental control (E-control) group, an additional group of locusts were inoculated with media only and considered the non-infected control. Groups 1, 2, 3, and 4 were infected as described in materials and methods. Fecal output per group was weighted daily for up to 7 days pi. Total fecal output was divided by the number of living locusts for every day. There was non-significant increase in fecal output one day after infection, followed by significant decrease until day 7 after infection, with $p$-value $0.03,0.04,0.05$, and 0.03 for group 1, 2, 3, and 4, respectively. Data was compared using paired t-test (with $p$-value $<0.05$ as significant). Results are presented as means from three independent experiments. 


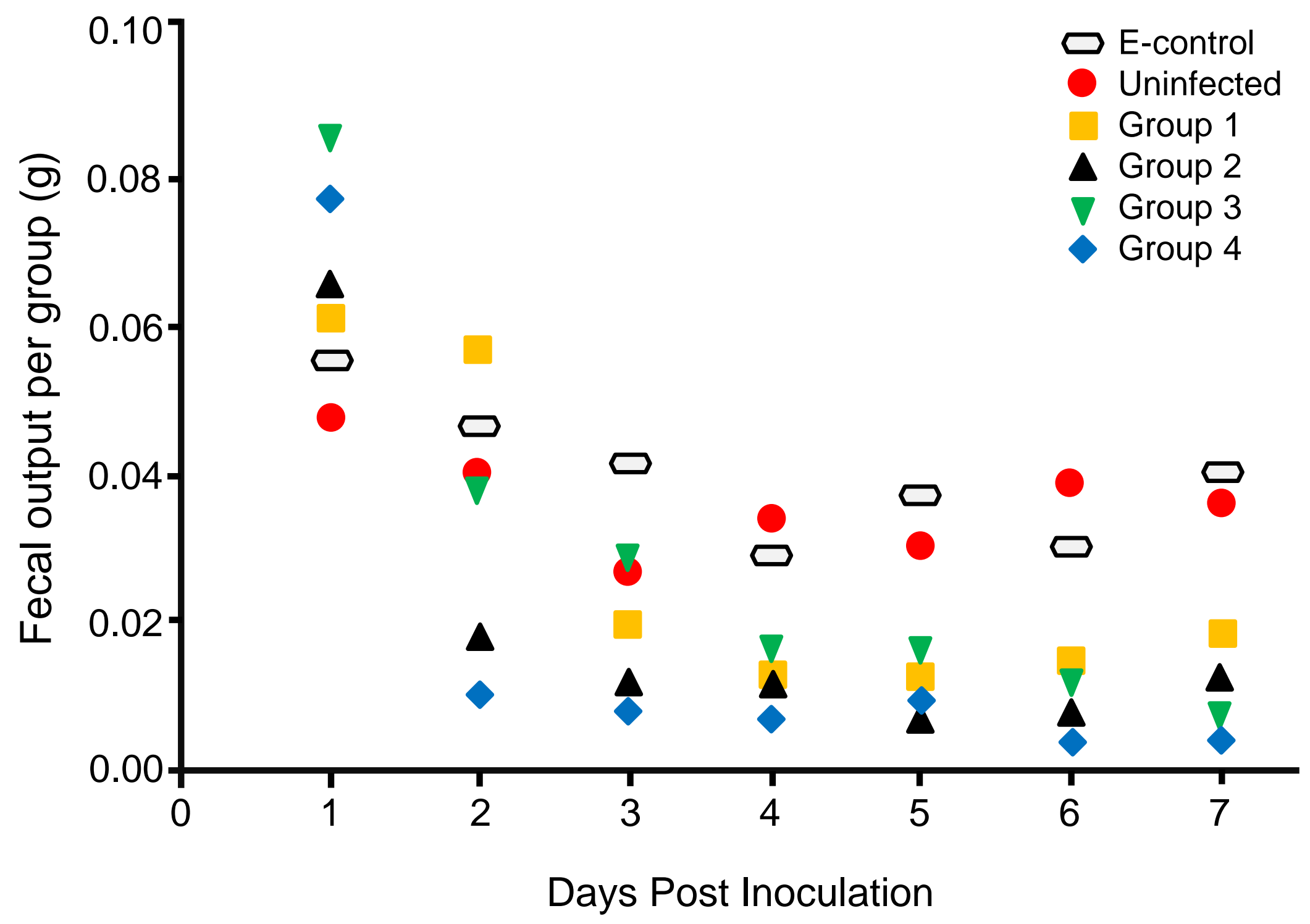




\section{Figure 4}

Representative micrographs of Neospora caninum-infected locust brains.

Locusts were injected with $10^{6} \mathrm{~N}$. caninum and their brains were dissected out at 5 days post-infection. Subsequently, the brains were sectioned and stained with haematoxylin and eosin. Infected locusts showed $N$. caninum triggered inflammatory response (arrows) in the brain tissue (A). No parasite was detected in the brain (A) or in the fat body surrounding the brain of (B). Magnification $\times 400$.
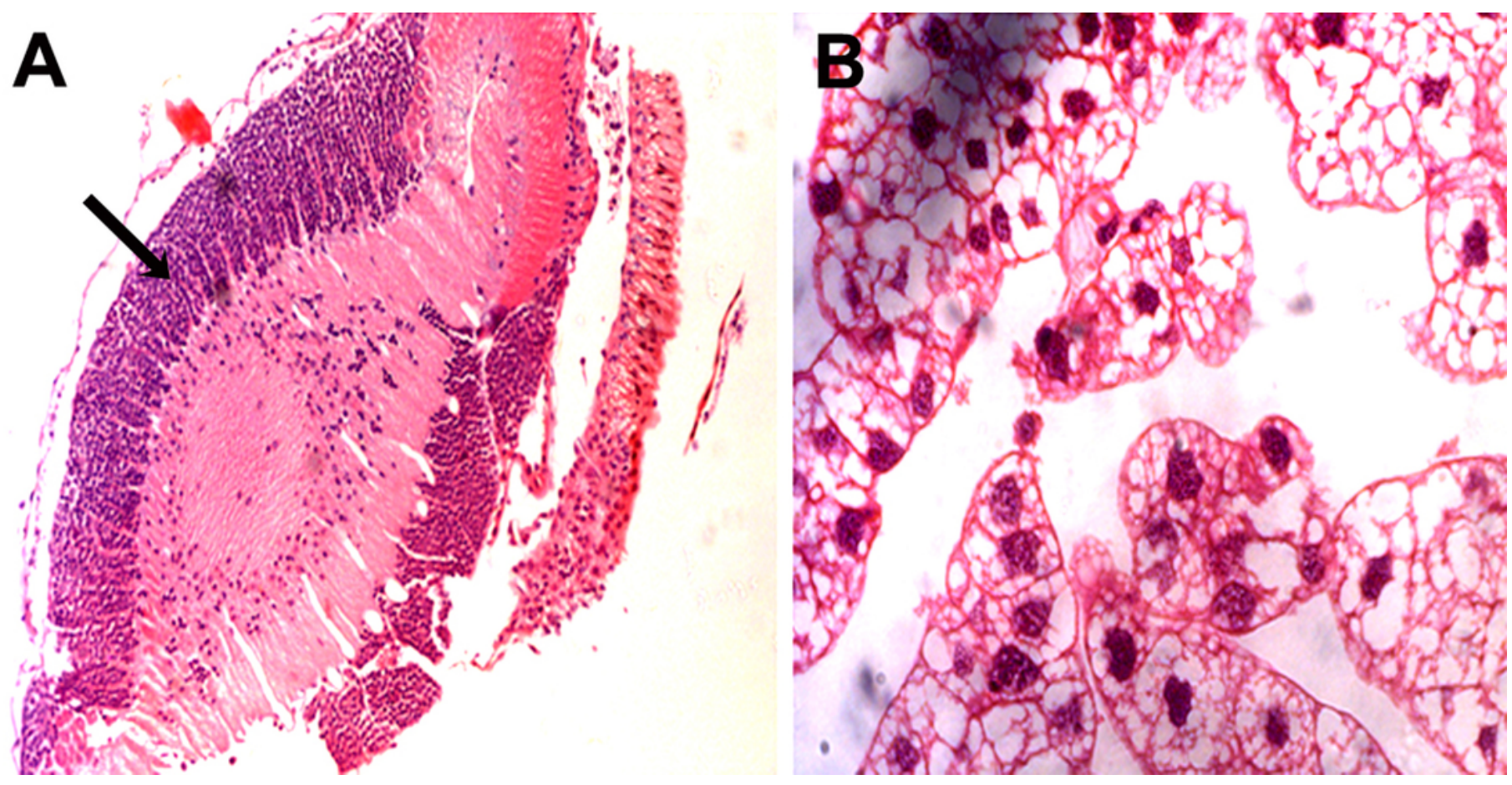


\section{Figure 5}

PCR amplification of the Neospora caninum-specific Nc5 region (Np21/Np6).

Amplification of DNA extracts from brains of locusts experimentally infected with $N$. caninum showed the presence of genetic evidence of $N$. caninum in the brain of locusts from day 1 (d1) to day 5 (d5) pi. M: 100-bp molecular size marker; Lane 1: positive control represent DNA extracted from $\sim 3 \times 10^{6}$ tachyzoites; lanes $\mathrm{d} 1$ to $\mathrm{d} 5$ : $\mathrm{N}$. caninum in brain $\mathrm{d} 1$ to $\mathrm{d} 5$.

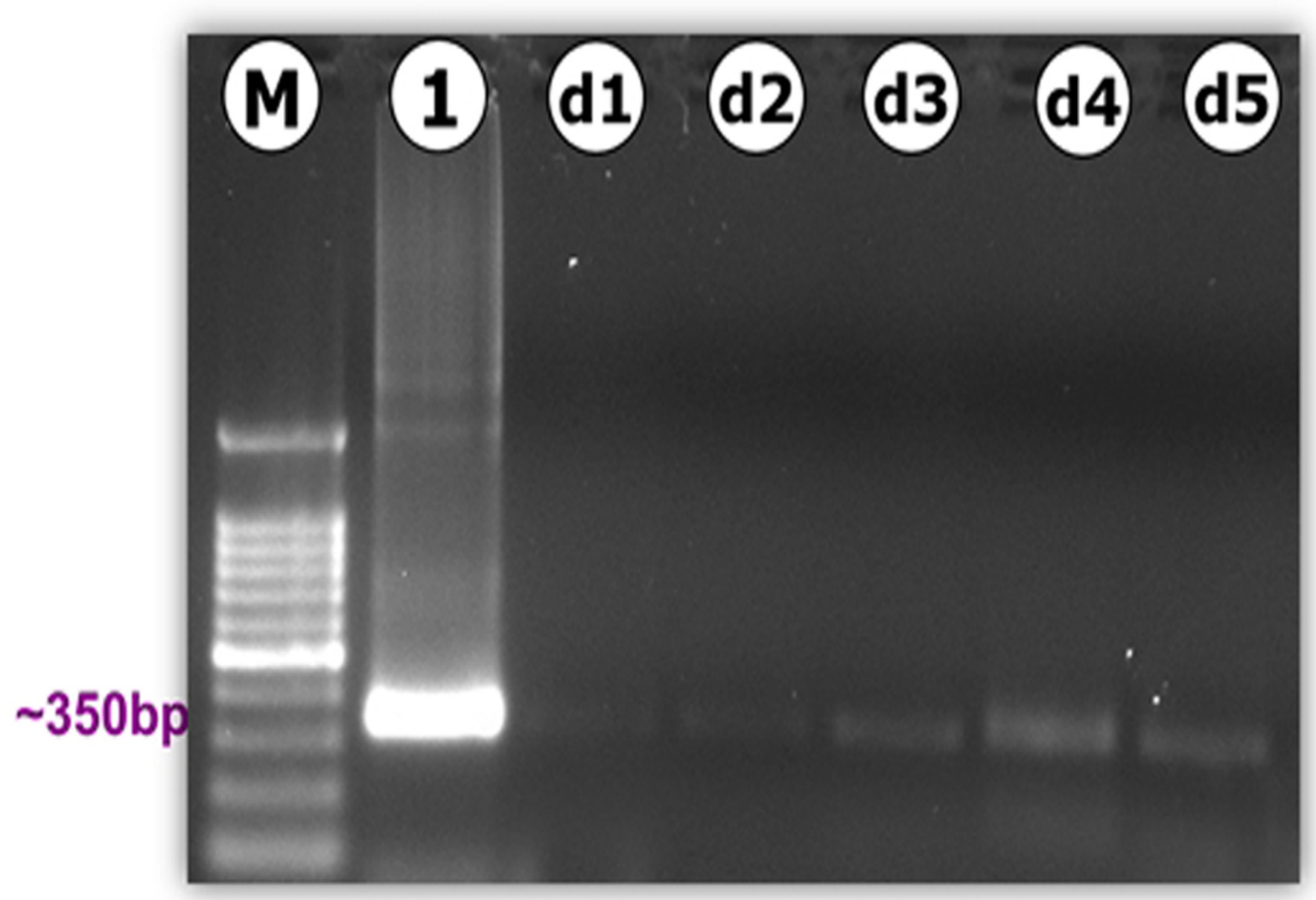




\section{Figure 6}

Raman spectroscopic imaging of tachyzoites.

The top panel shows bright field (A) and corresponding Raman (B) image of $N$. caninum tachyzoites derived from locust's brain. The bottom panel shows bright field (C) and corresponding Raman (D) image of N. caninum tachyzoites derived from culture. Bar applies to all figures, $5 \mu \mathrm{m}$.

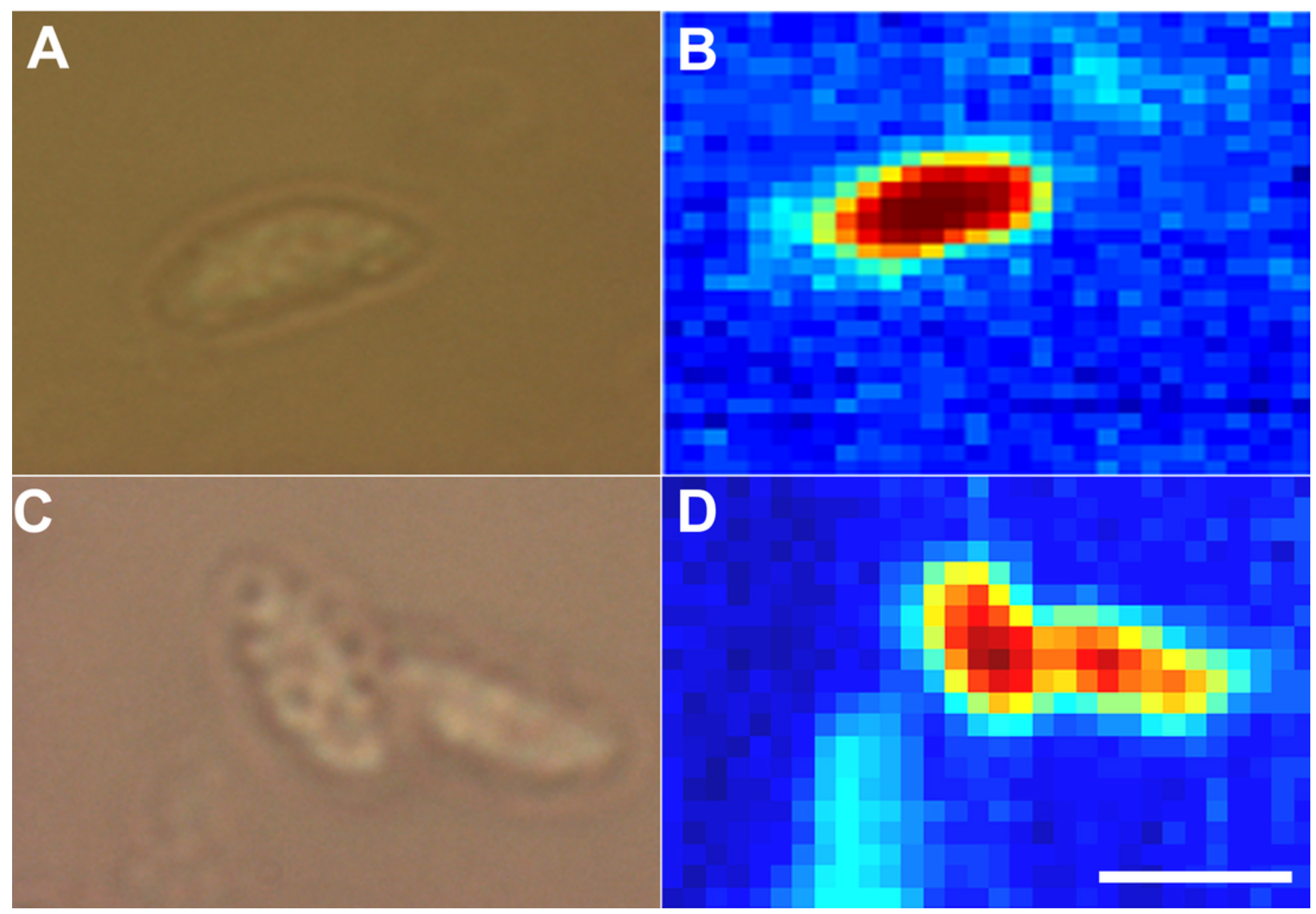




\section{Figure 7}

Comparative profiling of chemical structure of tachyzoites ofNeospora caninumfrom locust-derived and original isolates using Raman spectroscopy.

(A) Comparative Raman spectra of tachyzoites of $N$. caninumculture-derived (blue) and locust brain-derived isolates (green) in the region from 700 to $1700 \mathrm{~cm}^{-1}$.(B) Principal component analysis score plots in the plane of principal components $3 \mathrm{vs.} \mathrm{principal} \mathrm{component} 1$ for samples tested. Each dot represents a chemical molecule and the dots are colored according to the biological group the sample belongs to. Blue dots indicateN. caninumculture-derived isolate and green dots indicate locust brain-derived isolate. Minor (green dots at the top), but non-significant differences were present between the chemical profile of each isolate. Raman spectra of locust-derived isolate are less scatter (i.e. less variation in their structures) compared to spectra of culture-derived isolate. 

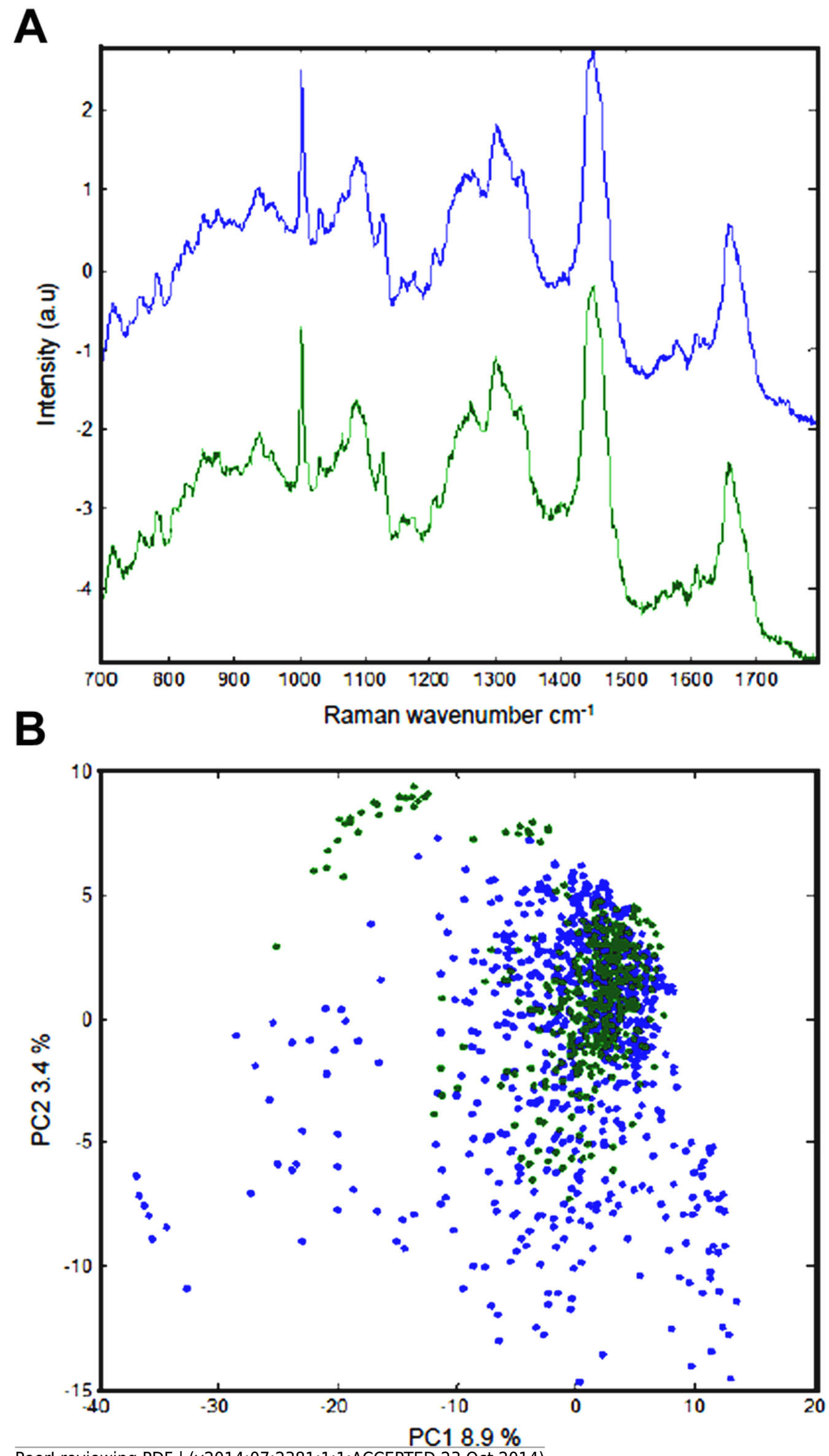


\section{Figure 8 (on next page)}

Heat map of differentially expressed lipids in locust brain.

Unsupervised two-dimensional hierarchical clustering of the 7 fatty acids that showed fold change differences between infected locust groups ( $n=5$ locusts) and their adjacent control daily for 5 days post infection with Neospora caninum. The heat map of differentially expressed lipids based on clustering is shown in the figure. Each column represents a lipid species and each row represents a locust group. Red colour indicates lipids that were upregulated and yellow color indicates lipids that were downregulated. Orange indicates lipids whose level is unchanged in infected locust's brain as compared to normal. A significant discriminative power between the infected and control samples of locust's brain was evident. Samples are identified by a three-part code: "F/C (infected/controls)"."Time point"."Replicate number". Fatty acids are reordered after applying a hierarchical clustering to their profiles. Hierarchical clustering of the rows and columns highlights groups of significantly correlated infection and lipids. 


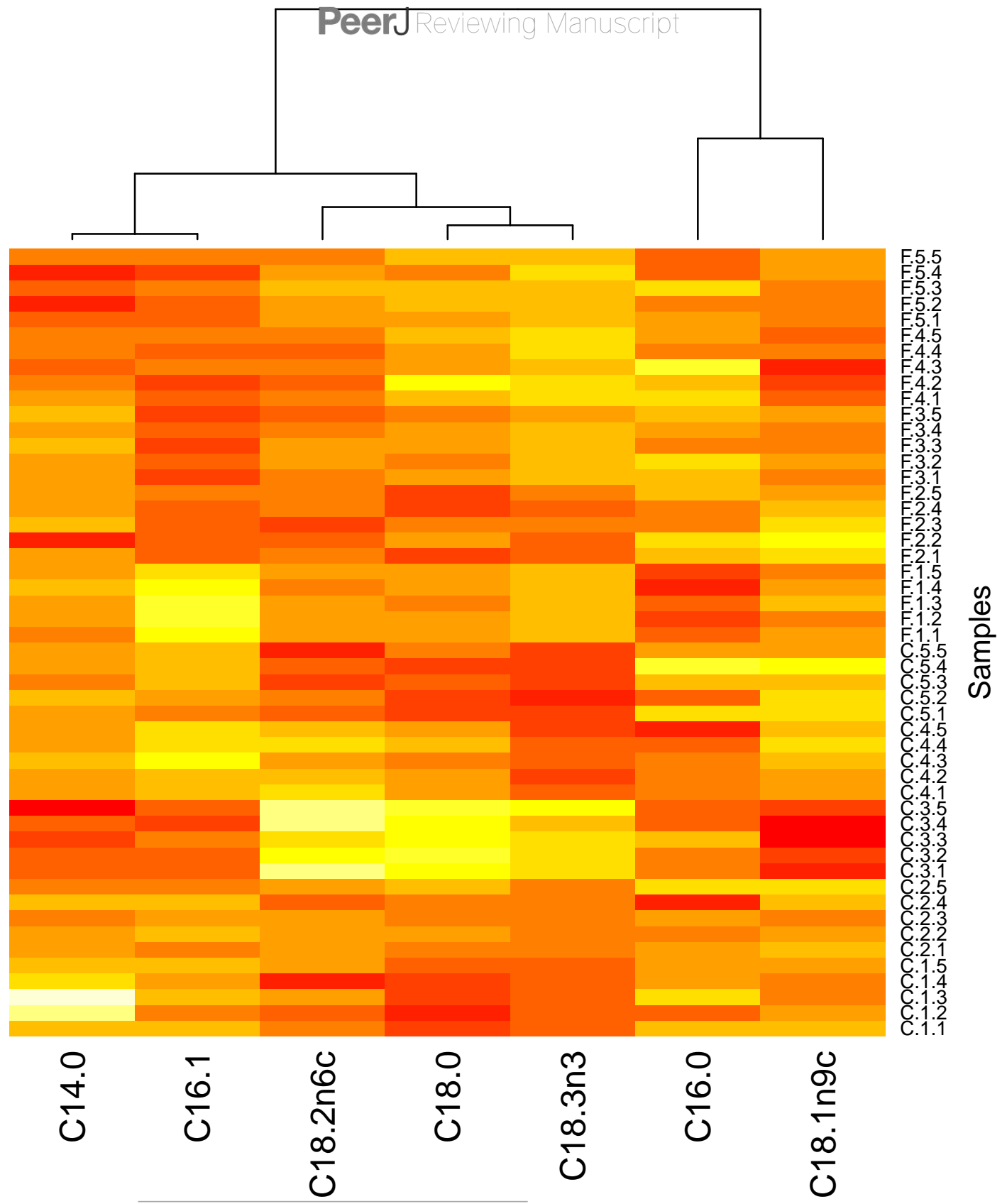


Figure 9 (on next page)

Volcano plot representation of the microarray data showing both significantly expressed transcripts and magnitude of change.

Negative $\log 10 \mathrm{p}$-value on $\mathrm{y}$ axis indicates the significance of each gene, and the fold change (log base 2) mean expression difference on the $x$ axis. Each gene is represented by a dot. Data are representative of three hybridizations per group. 


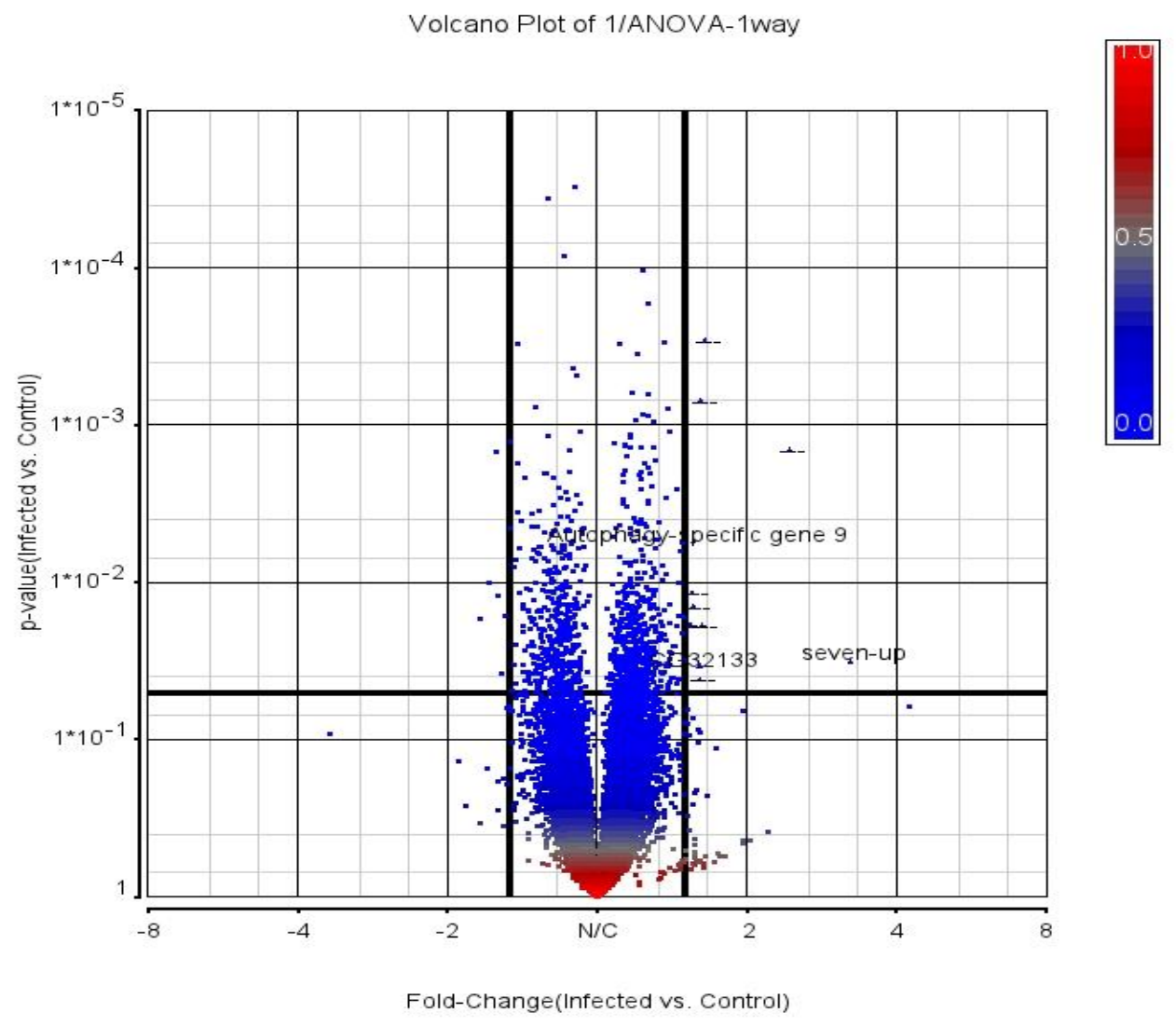


Figure 10 (on next page)

Hierarchical clustering of significantly expressed genes of three infected vs three control locusts.

The heat map shows two relatively distinct clusters of highly differentially expressed transcripts obtained from pairwise comparison between infected vs. control locust groups. Each row represents each sample tested and each column represents a single probeset (gene). On the hierarchical tree at the left side of the diagram, the upper half (red) indicates the control samples and the lower half (orange) indicates the infected samples. Relative gene expression is color represented: red is higher-level expression relative to the sample mean, blue is relatively lower-level expression, grey is no-change. The 11 probesets/genes in the upper right quadrant of the cluster map are genes that decreased upon infection relative to the control samples (shown in the lower right quadrant). The 6 probes/genes in the left upper quadrant were genes that were increased in control samples relative to infected samples (in the lower left quadrant). 


\section{PeerJ Reviewing Manuscript}

Hierarchical Clustering
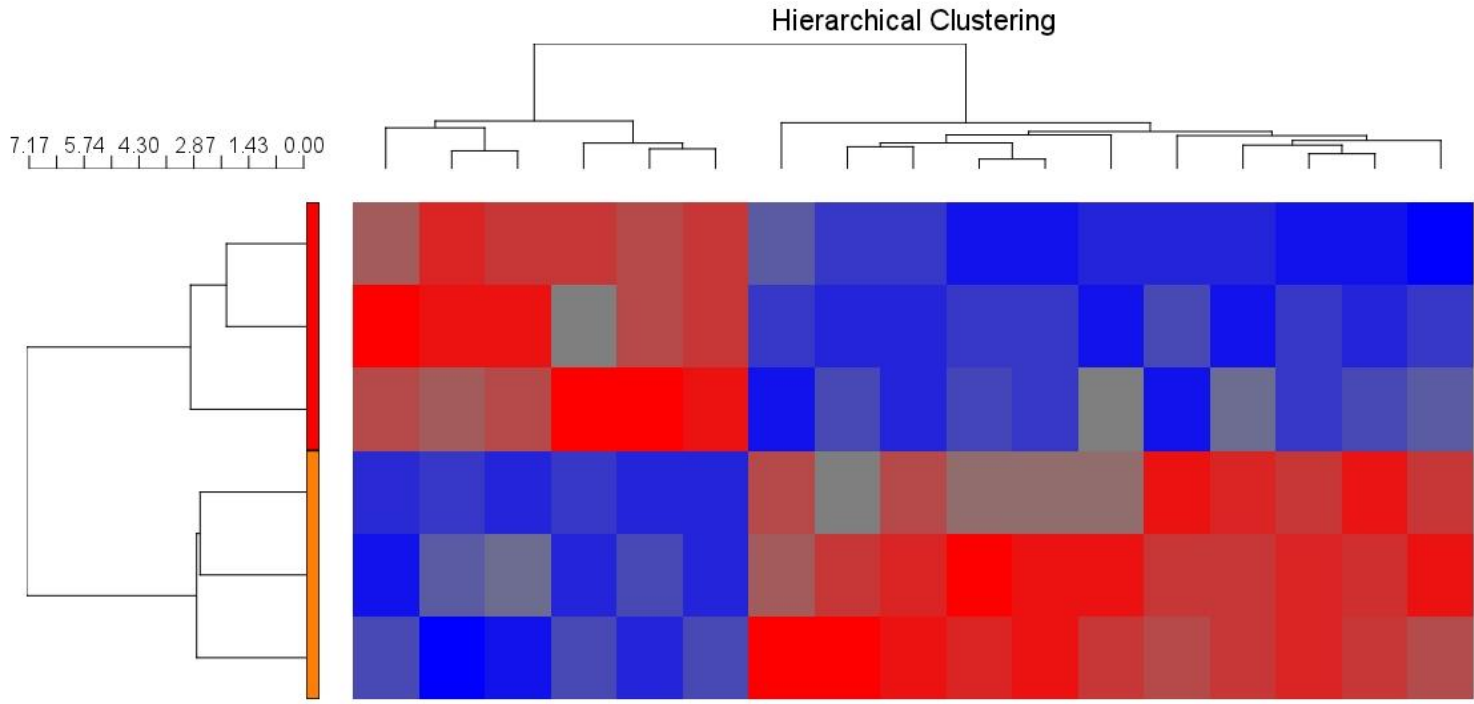

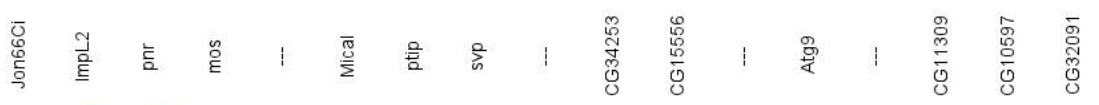

Treatment $\square$ Control $\square$ Infected

1.64 


\section{Table $\mathbf{1}$ (on next page)}

List of the top differentially expressed genes with the highest fold differences between Neospora caninum-infected locusts and uninfected controls. 


\section{PeerJ Reviewing Manuscript}

Table 1 List of the top differentially expressed genes with the highest fold differences between Neospora caninum-infected locusts and uninfected controls

\begin{tabular}{|c|c|c|c|c|}
\hline Probeset ID & $\begin{array}{l}\text { Gene } \\
\text { Symbol }\end{array}$ & Gene function & p-value & $\begin{array}{l}\text { Fold } \\
\text { difference }^{\text {a }}\end{array}$ \\
\hline \multicolumn{5}{|c|}{$\begin{array}{l}\text { Upregulated gene expression in association with infection. Genes with increased } \\
\text { expression in brain of infected locusts vs non-infected locusts }\end{array}$} \\
\hline 1628992_at & CG34253 & $\begin{array}{l}-- \\
-1\end{array}$ & 0.0184845 & 1.53941 \\
\hline 1623064_at & --- & --- & 0.0115445 & 1.55291 \\
\hline 1626577_at & CG15556 & --- & 0.0142076 & 1.56118 \\
\hline 1626033_at & Atg9 & Autophagy-specific gene 9 & 0.00543545 & 1.55952 \\
\hline 1626376_at & ptip & CG32133 & 0.0333639 & 1.60544 \\
\hline 1635717_x_at & --- & --- & 0.0410724 & 1.60703 \\
\hline 1629711_at & CG10597 & --- & 0.00069658 & 1.61358 \\
\hline 1640991_at & CG32091 & --- & 0.0186437 & 1.62449 \\
\hline 1632924_at & --- & --- & 0.00142772 & 2.43486 \\
\hline 1635192_at & svp & seven-up & 0.0317402 & 3.22575 \\
\hline
\end{tabular}

Downregulated gene expression in association with infection. Genes with decreased expression in brain of infected locusts vs non-infected locusts

\begin{tabular}{|c|c|c|c|c|}
\hline 1625538_at & Jon66Ci & $\begin{array}{l}\text { serine-type endopeptidase } \\
\text { activity }\end{array}$ & 0.0163373 & -1.5279 \\
\hline 1640434_at & mos & $\begin{array}{ll}-- \\
\end{array}$ & 0.0376856 & -1.55319 \\
\hline 1636202_s_at & ImpL2 & $\begin{array}{l}\text { Ecdysone-inducible gene L2 } \\
\text { InR signalling pathway }\end{array}$ & 0.0121551 & -1.58749 \\
\hline & & & 0.0014925 & \\
\hline 1636412_at & Mical & --- & 8 & -1.60216 \\
\hline 1630146_at & --- & $\begin{array}{ll}-- \\
--\end{array}$ & 0.0101685 & -1.65487 \\
\hline 1634370_a_at & $\begin{array}{l}\text { Panner } \\
(P n r)\end{array}$ & $\begin{array}{l}\text { Zinc-finger transcription } \\
\text { factor of the GATA family } \\
\text { (involved in several } \\
\text { embryogenesis processes and } \\
\text { imaginal development) }\end{array}$ & 0.0171417 & -1.72354 \\
\hline
\end{tabular}




\section{PeerJ Reviewing Manuscript}

${ }^{a}$ mRNA expression relative to uninfected controls, as determined by Microarray analysis, with 3 locusts per group. No difference between groups, 1.00. Genes with unknown function are indicated by (---). 